\title{
On Optimal Truncated Biharmonic Current Waveforms for Class-F and Inverse Class-F Power Amplifiers
}

\author{
Anamarija Juhas, ${ }^{1}$ Stanisa Dautovic, ${ }^{1}$ and Ladislav A. Novak $^{2}$ \\ ${ }^{1}$ Department of Power, Electronics and Communication Engineering, Faculty of Technical Sciences, University of Novi Sad, \\ Trg D. Obradovica 6, 21000 Novi Sad, Serbia \\ ${ }^{2}$ Computer Science Department, Indiana University Bloomington, 150 S. Woodlawn Ave., Bloomington, IN 47405, USA
}

Correspondence should be addressed to Ladislav A. Novak; ladnovak@indiana.edu

Received 4 October 2016; Revised 25 January 2017; Accepted 1 February 2017; Published 16 March 2017

Academic Editor: Alessandro Lo Schiavo

Copyright (c) 2017 Anamarija Juhas et al. This is an open access article distributed under the Creative Commons Attribution License, which permits unrestricted use, distribution, and reproduction in any medium, provided the original work is properly cited.

In this paper, two-parameter families of periodic current waveforms for class-F and inverse class-F power amplifiers (PAs) are considered. These waveforms are obtained by truncating cosine waveforms composed of dc component and fundamental and either second $(k=2)$ or third $(k=3)$ harmonic. In each period, waveforms are truncated to become zero outside of a prescribed interval (so-called conduction angle). The considered families of waveforms include both discontinuous and continuous waveforms. Fourier series expansion of truncated waveform contains an infinite number of harmonics, although a number of harmonics may be missing. Taking into account common assumptions that for class-F PA the third $(n=3)$ harmonic is missing in current waveform and for inverse class-F PA the second $(n=2)$ harmonic is missing in current waveform, we consider the following four cases: (i) $n=k=3$, (ii) $n=3, k=2$, (iii) $n=k=2$, and (iv) $n=2, k=3$. We show that, in each of these cases, current waveform enabling maximal efficiency (optimal waveform) of class-F and inverse class-F PA is continuous for all conduction angles of practical interest. Furthermore, we provide closed-form expressions for parameters of optimal current waveforms and maximal efficiency of class-F (inverse class-F) PA in terms of conduction angle only. Two case studies of practical interest for PA design, involving suboptimal current waveforms, along with the results of nonlinear simulation of inverse class-F PA, are also presented.

\section{Introduction}

There is continuous interest in class-F PA and its dual inverse class-F PA (see, e.g., [1-8]). Finding optimal and suboptimal nonnegative waveforms for PAs also attracted substantial interest within the research community (see, e.g., [5, 9-16]) and can be regarded as a part of the so-called waveform engineering $[15,16]$.

In this paper, we consider a two-parameter model of periodic current waveform, defined within fundamental period as

$$
\begin{aligned}
& i_{(k)}(\tau) \\
& = \begin{cases}I_{\mathrm{P}}\left(A_{0(k)}+\cos \tau-A_{k} \cos k \tau\right), & |\tau| \leq \tau_{p}, \\
0, & \tau_{p}<|\tau| \leq 180^{\circ},\end{cases}
\end{aligned}
$$

where $\tau$ stands for $\omega t, 0<2 \tau_{p}<360^{\circ}$ is conduction angle, $A_{0(k)}$ and $A_{k}$ are parameters, $I_{\mathrm{P}}>0$ is constant, and $k=2$ or $k=3$. This family includes both continuous and discontinuous waveforms. Waveform of type $i_{(k)}(\tau)$ is a truncated biharmonic current waveform.

The current waveform of type (1) is an even function and therefore its Fourier series expansion contains dc component and cosine terms. Coefficients of the Fourier series expansion can be expressed as

$$
\begin{gathered}
I_{\mathrm{dc}(k)}=\frac{I_{\mathrm{P}} \tau_{p}}{\pi}\left[A_{0(k)}+\operatorname{sinc} \tau_{p}-A_{k} \operatorname{sinc} k \tau_{p}\right], \\
I_{n(k)}=\frac{I_{\mathrm{P}} \tau_{p}}{\pi}\left\{2 A_{0(k)} \operatorname{sinc} n \tau_{p}+\operatorname{sinc}(n-1) \tau_{p}\right. \\
\quad+\operatorname{sinc}(n+1) \tau_{p} \\
\left.-A_{k}\left[\operatorname{sinc}(k-n) \tau_{p}+\operatorname{sinc}(k+n) \tau_{p}\right]\right\},
\end{gathered}
$$


where $n \geq 1$ and $\operatorname{sinc}(\cdot)$ denotes unnormalized sinc function $\operatorname{sinc} x=(\sin x) / x$. Coefficients of fundamental harmonic $(n=1)$ and $k$ th harmonic $(n=k)$ can be also obtained from (3) by using that $\operatorname{sinc}(0)=1$. Notice that change of parameter $A_{k}$ and/or $A_{0(k)}$ causes the change of the whole harmonic content of waveform of type (1).

Corresponding voltage waveform of PA is assumed to be of the form

$$
v_{(n)}(\tau)=V_{\mathrm{dc}}\left[1-\gamma_{V(n)}\left(\cos \tau-a_{n} \cos n \tau\right)\right]
$$

where $V_{\mathrm{dc}}>0, \gamma_{V(n)}>0$, and $n=2$ or $n=3$.
A number of existing models of current waveforms (both continuous and discontinuous) can be embedded in model (1), including the most used continuous model of current waveform for classical PA operation (see, e.g., [5]):

$$
i_{\text {classic }}(\tau)= \begin{cases}I_{\mathrm{P}}\left(\cos \tau-\cos \tau_{p}\right), & |\tau| \leq \tau_{p} \\ 0, & \tau_{p} \leq|\tau| \leq 180^{\circ}\end{cases}
$$

Model (5) can be obtained directly from (1) by setting $A_{0(k)}=$ $-\cos \tau_{p}$ and $A_{k}=0$.

Another widely considered continuous model of current waveform is one-parameter model of type (see, e.g., [5])

$$
i_{(k) \mathrm{cont}}(\tau)= \begin{cases}I_{\mathrm{P}}\left[\cos \tau-\cos \tau_{p}-A_{k}\left(\cos k \tau-\cos k \tau_{p}\right)\right], & |\tau| \leq \tau_{p}, \\ 0, & \tau_{p} \leq|\tau| \leq 180^{\circ}\end{cases}
$$

which can be also obtained from (1) by setting the value of parameter $A_{0(k)}$ to

$$
A_{0(k)}=-\cos \tau_{p}+A_{k} \cos k \tau_{p} .
$$

Typically, continuous current waveform of type (6) is paired with voltage waveform of type (4) with $n=k$ for biharmonic mode of PA operation [5, 17]. In [17], the value of parameter $A_{k}$ (for $k=2$ and $k=3$ ) is obtained via optimization of efficiency, subject to the constraint that $k$ th harmonic coefficient $I_{k(k)}$ is nonpositive. A special case of biharmonic mode with $A_{k}=a_{k}$ is also analyzed in $[5,17]$.

The two-parameter model of continuous current waveform for PA with $n=k=2$ has been considered in [18]. This model can be obtained from (1) by setting $k=2, I_{\mathrm{P}}=$ $I_{\text {max }} f\left(h_{2}, c\right), A_{0(k)}=c / f\left(h_{2}, c\right)$, and $A_{k}=-h_{2}$.

The one-parameter model of discontinuous current waveform is used in [3]. The authors of [3] considered case $n=k=$ 3 in the context of class-F PA and case $n=k=2$ in the context of inverse class-F PA. Current waveform proposed in [3] for class-F can be obtained from (1) by setting $k=3, A_{0(k)}=$ $-\cos \tau_{p}, A_{k}=\beta_{F} / I_{\mathrm{P}}$, and $I_{\mathrm{P}}=\left(1+\beta_{F} / I_{\max }\right) I_{D}$. Furthermore, parameter $\beta_{F}$ is determined from the condition that the third harmonic is missing in current waveform. The same model of current waveform is also used in [19] in the context of continuous class-F PA.

On the other hand, for inverse class-F, the one-parameter model of discontinuous current waveform proposed in [3] can be obtained from (1) by setting $k=2, A_{0(k)}=$ $-\cos \tau_{p}, A_{k}=\beta_{F^{-1}} / I_{\mathrm{P}}$, and $I_{\mathrm{P}}=\left(1+\beta_{F} / I_{\max }\right) I_{D}$. In this context, parameter $\beta_{F^{-1}}$ is obtained from the condition that the second harmonic is missing in current waveform.

For class-F PA, we use the common assumption that the third harmonic in current waveform is missing, $I_{3(k)}=0$ (see, e.g., $[2,3,7])$. In this case, the corresponding voltage waveform is of type (4) for $n=3$. Also, for inverse class-F PA, we use the common assumption that the second harmonic in current waveform is missing, $I_{2(k)}=0$ (see, e.g., $[2,3]$ ). The corresponding voltage waveform is of type (4) for $n=2$.
In what follows, current waveforms of type (1) satisfying condition $I_{n(k)}=0$ are denoted by $i_{\left(k, I_{n}=0\right)}(\tau)$. In Sections $2-4$, we consider current waveform $i_{\left(k, I_{n}=0\right)}(\tau)$ of type (1) for $k \in\{2,3\}$ and $n \in\{2,3\}$. To be more specific, we consider the following four cases:
(i) $n=k=3$,
(ii) $n=3$ and $k=2$,
(iii) $n=k=2$,
(iv) $n=2$ and $k=3$.

Cases (i) and (ii) correspond to class-F mode of PA operation, whereas cases (iii) and (iv) correspond to inverse class-F mode. To the best of our knowledge, cases (ii) and (iv) are not widely explored in waveform modeling for PA design.

The efficiency $\eta$ of PA can be expressed via basic waveform parameters $\gamma_{I}$ and $\gamma_{V}$ as $\eta=\gamma_{I} \gamma_{V} / 2$, provided that parameter $\gamma_{I}\left(\gamma_{V}\right)$ is equal to the quotient of fundamental harmonic amplitude and dc component of the current (voltage) waveform (see, e.g., [1]). In class-F and inverse class-F $\mathrm{PA}$, a higher harmonic component could appear in at most one of the waveforms in current-voltage pair (see, e.g., [1]). This fact implies that current and voltage waveforms can be optimized independently for class-F and inverse class-F PA. Therefore, nonnegative current waveform of type $i_{\left(k, I_{n}=0\right)}(\tau)$ that ensures maximal efficiency (optimal waveform) of classF PA or inverse class-F PA is a waveform of type $i_{\left(k, I_{n}=0\right)}(\tau)$ with maximal parameter $\gamma_{I}$.

For all cases (i)-(iv), in Section 2, we prove that current waveforms of type (1) enabling maximal efficiency are continuous for all conduction angles of practical interest for class- $\mathrm{F}$ and inverse class-F PA. Closed-form expressions for parameters of such waveforms as functions of conduction angle are also provided in Section 2. In Section 3, an independent numerical verification of the values of parameters of optimal current waveform is described. In Section 4, we consider efficiency of class-F and inverse class-F PA, using the results obtained in Section 2. In Section 4.1, maximal efficiency of 
class-F PA with optimal current waveforms for $k=2$ or $k=3$ is provided. Section 4.2 is devoted to inverse class-F PA with optimal current waveforms for $k=2$ or $k=3$. In Section 5, we consider continuous current waveforms of type (6) satisfying relaxed condition $I_{n(k)} \leq 0$ for $k \in\{2,3\}$ and $n \in\{2,3\}$, instead of $I_{n(k)}=0$ used in the context of class-F and inverse class-F PA. Two case studies involving suboptimal current waveforms for class- $\mathrm{F}$ and inverse class- $\mathrm{F}$ are also presented in this section. As a practical validation of the proposed approach, comparisons of the results derived in this paper with the result of nonlinear simulation of inverse classF PA with CGH40010F HEMT are provided in Section 6.

\section{Optimal Current Waveform of Class-F and Inverse Class-F PA}

In this section, current waveforms $i_{\left(k, I_{n}=0\right)}(\tau)$ of type (1) that satisfy condition $I_{n(k)}=0$ for $n \in\{2,3\}$ and $k \in\{2,3\}$ are considered. In what follows, we prove that current waveform $i_{\left(k, I_{n}=0\right)}(\tau)$, which in pair with voltage waveform of type (4) enables maximal efficiency, is continuous for all conduction angles of practical interest for class- $F$ and inverse class-F PA (i.e., for those conduction angles listed in Table 1). In this section, we also provide closed-form expressions for parameters of such waveforms.
TABLE 1: Conduction angles for which the optimal waveform of type $i_{\left(k, I_{n}=0\right)}(\tau)$ is continuous.

\begin{tabular}{llcc}
\hline Case & $n$ & $k$ & $\begin{array}{c}\text { Conduction } \\
\text { angle } 2 \tau_{p}\end{array}$ \\
\hline (i) & 3 & 3 & $2 \tau_{p} \geq 97.4^{\circ}$ \\
(ii) & 3 & 2 & $2 \tau_{p} \geq 96.4^{\circ}$ \\
(iii) & 2 & 2 & $2 \tau_{p} \geq 146.4^{\circ}$ \\
(iv) & 2 & 3 & $2 \tau_{p} \geq 151.1^{\circ}$ \\
\hline
\end{tabular}

In what follows, we first prove that optimal waveform is continuous.

As it has been mentioned earlier, Fourier coefficient of $n$th harmonic of current waveform of type (1) can be expressed in form (3). Furthermore, condition $I_{n(k)}=0$ implies that parameter $A_{k}$ is equal to

$$
\begin{aligned}
& A_{k\left(I_{n}=0\right)} \\
& \quad=\frac{2 A_{0(k)} \operatorname{sinc} n \tau_{p}+\operatorname{sinc}(n-1) \tau_{p}+\operatorname{sinc}(n+1) \tau_{p}}{\operatorname{sinc}(k-n) \tau_{p}+\operatorname{sinc}(k+n) \tau_{p}} .
\end{aligned}
$$

From (2) and (3), it follows that basic waveform parameter $\gamma_{I\left(k, I_{n}=0\right)}=I_{1\left(k, I_{n}=0\right)} / I_{\mathrm{dc}\left(k, I_{n}=0\right)}$ of waveform $i_{\left(k, I_{n}=0\right)}(\tau)$ reads

$$
\gamma_{I\left(k, I_{n}=0\right)}=\frac{2 A_{0(k)} \operatorname{sinc} \tau_{p}+1+\operatorname{sinc} 2 \tau_{p}-A_{k\left(I_{n}=0\right)}\left[\operatorname{sinc}(k-1) \tau_{p}+\operatorname{sinc}(k+1) \tau_{p}\right]}{A_{0(k)}+\operatorname{sinc} \tau_{p}-A_{k\left(I_{n}=0\right)} \operatorname{sinc} k \tau_{p}},
$$

where $A_{k\left(I_{n}=0\right)}$ is given by (8). For the prescribed conduction angle, parameter $A_{k\left(I_{n}=0\right)}$ is a function of $A_{0(k)}$ only, which implies that $\gamma_{I\left(k, I_{n}=0\right)}$ is also a function of $A_{0(k)}$ only. First derivative of $\gamma_{I\left(k, I_{n}=0\right)}$ with respect to $A_{0(k)}$ can be expressed in the following form:

$$
\frac{d \gamma_{I\left(k, I_{n}=0\right)}}{d A_{0(k)}}=\left(\frac{I_{\mathrm{P}} \tau_{p}}{\pi I_{\mathrm{dc}\left(k, I_{n}=0\right)}}\right)^{2} f_{(n, k)}\left(\tau_{p}\right),
$$

where $f_{(n, k)}\left(\tau_{p}\right)$ is a function of $\tau_{p}$ :

$$
\begin{aligned}
& f_{(n, k)}\left(\tau_{p}\right)=\left[\operatorname{sinc}(k-n) \tau_{p}+\operatorname{sinc}(k+n) \tau_{p}\right]^{-1} \\
& \quad \times\left\{\left[\operatorname{sinc}(k-1) \tau_{p}+\operatorname{sinc}(k+1) \tau_{p}\right.\right. \\
& \left.\quad-2 \operatorname{sinc} \tau_{p} \operatorname{sinc} k \tau_{p}\right] \times\left[\operatorname{sinc}(n-1) \tau_{p}\right. \\
& \left.+\operatorname{sinc}(n+1) \tau_{p}-2 \operatorname{sinc} \tau_{p} \operatorname{sinc} n \tau_{p}\right] \\
& -\left[\operatorname{sinc}(k-n) \tau_{p}+\operatorname{sinc}(k+n) \tau_{p}-2 \operatorname{sinc} k \tau_{p}\right. \\
& \left.\left.\cdot \operatorname{sinc} n \tau_{p}\right] \times\left[1+\operatorname{sinc} 2 \tau_{p}-2 \operatorname{sinc}^{2} \tau_{p}\right]\right\} .
\end{aligned}
$$

It is easy to see that the sign of $d \gamma_{I\left(k, I_{n}=0\right)} / d A_{0(k)}$ depends on the sign of $f_{(n, k)}\left(\tau_{p}\right)$ only. Since $f_{(n, k)}\left(\tau_{p}\right)$ is negative for $n \in\{2,3\}, k \in\{2,3\}$, and $2 \tau_{p} \in\left(0,360^{\circ}\right)$ (see Figure 1 ), it immediately follows that $\gamma_{I\left(k, I_{n}=0\right)}$ decreases when $A_{0(k)}$ increases. Consequently, the highest value of $\gamma_{I\left(k, I_{n}=0\right)}$ (and the highest efficiency) is attained for minimal value of $A_{0(k)}$.

In order to be nonnegative, waveform $i_{\left(k, I_{n}=0\right)}(\tau)$ should satisfy condition $i_{\left(k, I_{n}=0\right)}\left( \pm \tau_{p}\right) \geq 0$, that is, $A_{0(k)} \geq-\cos \tau_{p}+$ $A_{k\left(I_{n}=0\right)} \cos k \tau_{p}$. For minimal value of $A_{0(k)}$ (see the above consideration), this condition reduces to equality. Therefore, $A_{0(k)}$ of optimal current waveform is equal to

$$
A_{0(k)}=-\cos \tau_{p}+A_{k\left(I_{n}=0\right)} \cos k \tau_{p}
$$

which further implies that optimal waveform of type $i_{\left(k, I_{n}=0\right)}(\tau)$ is continuous; that is, it is of type (6).

After substitution of (12) into (8) and solving the resulting equation, we obtain parameter $A_{k}$ of continuous waveform of type (6) that satisfies condition $I_{n(k)}=0$, which we denote by $A_{k\left(I_{n}=0\right) \text { cont }}$. This parameter is a function of conduction angle only:

$$
A_{k\left(I_{n}=0\right) \mathrm{cont}}=\frac{\operatorname{sinc}(n-1) \tau_{p}-\operatorname{sinc}(n+1) \tau_{p}}{k\left[\operatorname{sinc}(k-n) \tau_{p}-\operatorname{sinc}(k+n) \tau_{p}\right]} .
$$

Consequently, optimal current waveform of class-F PA (inverse class-F PA) is a continuous waveform of type (6) with parameter $A_{k}=A_{k\left(I_{n}=0\right) \text { cont }}, k \in\{2,3\}$, and $n=3(n=2)$. In what follows, we show that this waveform is nonnegative for the conduction angles listed in Table 1. 


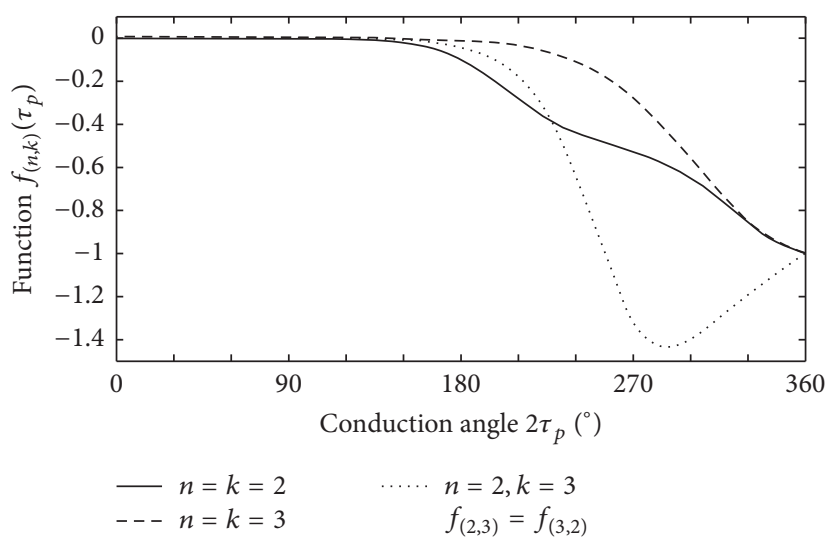

FIgURE 1: Functions $f_{(2,2)}\left(\tau_{p}\right)$ (solid line), $f_{(3,3)}\left(\tau_{p}\right)$ (dashed line), and $f_{(2,3)}\left(\tau_{p}\right)=f_{(3,2)}\left(\tau_{p}\right)$ (dotted line).

Let us first consider cases (ii) and (iii), that is, cases when $k=2$.
It is easy to show that continuous waveform of type (6) for $k=2$ can be expressed in factored form as

$$
i_{(k=2) \text { cont }}(\tau)= \begin{cases}I_{\mathrm{P}}\left(\cos \tau-\cos \tau_{p}\right)\left[1-2 A_{2}\left(\cos \tau+\cos \tau_{p}\right)\right], & |\tau| \leq \tau_{p} \\ 0, & \tau_{p} \leq|\tau| \leq 180^{\circ}\end{cases}
$$

Because $I_{\mathrm{P}}>0$ and $\cos \tau-\cos \tau_{p}>0$ for $|\tau|<\tau_{p}$, waveform of type $i_{(k=2) \text { cont }}(\tau)$ is nonnegative if and only if $1-2 A_{2}(\cos \tau+$ $\left.\cos \tau_{p}\right) \geq 0$ for $|\tau|<\tau_{p}$. By inspection of the first derivative of $1-2 A_{2}\left(\cos \tau+\cos \tau_{p}\right)$, it follows that it has only one critical point in interval $|\tau|<\tau_{p}$. Since this critical point is at $\tau=0$, it immediately follows that $i_{(k=2) \text { cont }}(\tau)$ is nonnegative provided that $1-2 A_{2}\left(1+\cos \tau_{p}\right) \geq 0$. This condition can be rewritten as

$$
A_{2} \leq A_{2 \max }
$$

where

$$
A_{2 \max }=\frac{1}{2\left(1+\cos \tau_{p}\right)}
$$

The dotted line in Figure 2 corresponds to $A_{2 \max }$.

Case (ii): $n=3$ and $k=2$. For $n=3$ and $k=2$, expression (13) can be simplified to

$$
A_{2\left(I_{3}=0\right) \operatorname{cont}}=\frac{5 \cos \tau_{p}}{4\left(1+4 \cos ^{2} \tau_{p}\right)}
$$

The solid line in Figure 2 corresponds to $A_{2\left(I_{3}=0\right) \text { cont }}$. It is easy to see that $\operatorname{sgn}\left[A_{2\left(I_{3}=0\right) \text { cont }}\right]=\operatorname{sgn}\left[\cos \tau_{p}\right]$. According to the above consideration, waveform $i_{(k=2) \text { cont }}(\tau)$ with $A_{2}=$ $A_{2\left(I_{3}=0\right) \text { cont }}$ is nonnegative if $A_{2\left(I_{3}=0\right) \text { cont }} \leq A_{2 \max }$. Substituting
(17) and (16) into $A_{2\left(I_{3}=0\right) \text { cont }} \leq A_{2 \max }$ and solving the resulting relation for $\tau_{p}$ lead to $\tau_{p} \geq \arccos (2 / 3) \approx 48.2^{\circ}$. Consequently, waveform $i_{(k=2) \text { cont }}(\tau)$ with $A_{2}=A_{2\left(I_{3}=0\right) \text { cont }}$ is nonnegative when $2 \tau_{p} \geq 96.4^{\circ}$ (third row in Table 1 ).

Case (iii): $n=2$ and $k=2$. Substitution of $n=2$ and $k=2$ into (13) leads to

$$
A_{2\left(I_{2}=0\right) \text { cont }}=\frac{8 \sin ^{2} \tau_{p}}{3\left(4 \tau_{p}-\sin 4 \tau_{p}\right)}
$$

The dashed line in Figure 2 corresponds to $A_{2\left(I_{2}=0\right) \text { cont }}$. It is easy to show that $A_{2\left(I_{2}=0\right) \text { cont }}>0$ for all $\tau_{p}\left(0<\tau_{p}<180^{\circ}\right)$. According to the above discussion, waveform $i_{(k=2) \text { cont }}(\tau)$ with $A_{2}=A_{2\left(I_{2}=0\right) \text { cont }}$ is nonnegative if $A_{2\left(I_{2}=0\right) \text { cont }} \leq A_{2 \max }$. Substituting (18) and (16) into $A_{2\left(I_{2}=0\right) \text { cont }} \leq A_{2 \max }$ and solving the resulting relation for $\tau_{p}$, we obtain $2 \tau_{p} \geq 146.4^{\circ}$. Consequently, waveform of type $i_{(k=2) \text { cont }}(\tau)$ with $A_{2}=$ $A_{2\left(I_{2}=0\right) \text { cont }}$ is nonnegative when $2 \tau_{p} \geq 146.4^{\circ}$ (fourth row in Table 1).

A number of shapes of current waveforms of type $i_{(k=2) \text { cont }}(\tau)$ satisfying $I_{n(k=2)}=0$ are presented in Figure 3 . Figure 3(a) corresponds to the case $n=3$ (case (ii)), whereas Figure 3(b) corresponds to the case $n=2$ (case (iii)).

Let us now consider cases (i) and (iv), that is, cases when $k=3$.

It is easy to show that continuous waveform of type (6) for $k=3$ can be expressed in factored form as

$$
i_{(k=3) \text { cont }}(\tau)= \begin{cases}I_{\mathrm{P}}\left(\cos \tau-\cos \tau_{p}\right)\left[1+3 A_{3}\left(1-\cos ^{2} \tau_{p}\right)-A_{3}\left(2 \cos \tau+\cos \tau_{p}\right)^{2}\right], & |\tau| \leq \tau_{p} \\ 0, & \tau_{p} \leq|\tau| \leq 180^{\circ}\end{cases}
$$




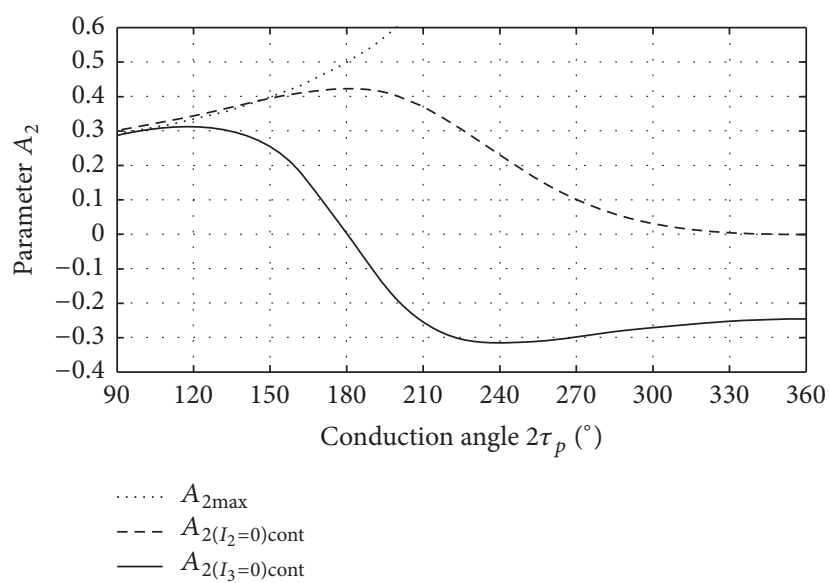

Figure 2: Parameter $A_{2}: A_{2 \max }$ (dotted line), $A_{2\left(I_{3}=0\right) \text { cont }}$ (solid line), and $A_{2\left(I_{2}=0\right) \text { cont }}$ (dashed line).

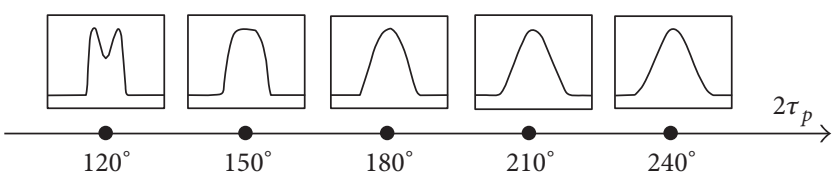

(a)

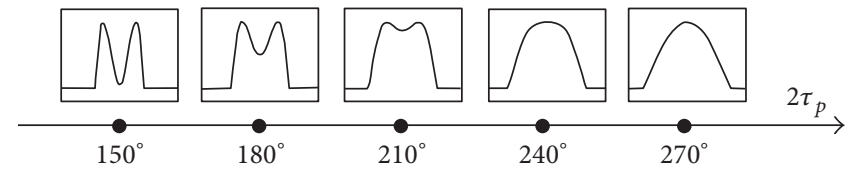

(b)

FIGURE 3: Shapes of current waveform of type $i_{(k=2) \text { cont }}(\tau)$ with (a) $A_{2}=A_{2\left(I_{3}=0\right) \text { cont }}$ and (b) $A_{2}=A_{2\left(I_{2}=0\right) \text { cont }}$.

Let us introduce an auxiliary waveform

$$
\begin{aligned}
g(\tau)= & 1+3 A_{3}\left(1-\cos ^{2} \tau_{p}\right) \\
& -A_{3}\left(2 \cos \tau+\cos \tau_{p}\right)^{2} .
\end{aligned}
$$

Because $I_{\mathrm{P}}>0$ and $\cos \tau-\cos \tau_{p}>0$ for $|\tau|<\tau_{p}$, waveform of type $i_{(k=3) \text { cont }}(\tau)$ is nonnegative if and only if $g(\tau) \geq 0$ for $|\tau|<\tau_{p}$. First derivative of $g(\tau)$ is equal to $g^{\prime}(\tau)=2 A_{3}\left(2 \cos \tau+\cos \tau_{p}\right) \sin \tau$. Therefore, critical points of $g(\tau)$ on interval $|\tau|<\tau_{p}$ are $\tau=0$ and $\tau=\tau_{c}$ satisfying $2 \cos \tau_{c}+\cos \tau_{p}=0$. Notice that relations $2 \cos \tau_{c}+\cos \tau_{p}=0$ and $\left|\tau_{c}\right|<\tau_{p}$ imply $\cos \tau_{p}<0$, that is, $\tau_{p}>90^{\circ}$. Values of $g(\tau)$ at critical points are $g(0)=1-A_{3}\left(1+2 \cos \tau_{p}\right)^{2}$ and $g\left(\tau_{c}\right)=1+3 A_{3}\left(1-\cos ^{2} \tau_{p}\right)$. Condition $g(0) \geq 0$ can be rewritten as

$$
A_{3} \leq A_{3 \max }
$$

where

$$
A_{3 \max }=\frac{1}{\left(1+2 \cos \tau_{p}\right)^{2}} .
$$

Also, condition $g\left(\tau_{c}\right) \geq 0$ can be rewritten as

$$
A_{3 \min } \leq A_{3} \text {, }
$$

where

$$
A_{3 \min }=\frac{-1}{3\left(1-\cos ^{2} \tau_{p}\right)} .
$$

From (24), it follows that $A_{3 \min }<-1 / 3$. Consideration of critical points of $g(\tau)$ leads to the conclusion that $i_{(k=3) \text { cont }}(\tau)$ is nonnegative if
(a) $-1 / 3 \leq A_{3} \leq A_{3 \max }$,
(b) $A_{3}<-1 / 3$ and $\tau_{p} \leq 90^{\circ}$,
(c) $A_{3 \min } \leq A_{3}<-1 / 3$ and $\tau_{p}>90^{\circ}$.

In what follows, we show that $A_{3\left(I_{3}=0\right) \text { cont }}>-1 / 3$ and $A_{3\left(I_{2}=0\right) \text { cont }}>-1 / 3$. Thus, both correspond to case (a), whereas cases (b) and (c) do not occur for waveforms of type $i_{(k=3) \text { cont }}(\tau)$ with $A_{3}=A_{3\left(I_{3}=0\right) \text { cont }}$ or $A_{3}=$ $A_{3\left(I_{2}=0\right) \text { cont }}$. Consequently, waveform $i_{(k=3) \text { cont }}(\tau)$ with $A_{3}=$ $A_{3\left(I_{3}=0\right) \text { cont }}\left(A_{3}=A_{3\left(I_{2}=0\right) \text { cont }}\right)$ is nonnegative if $A_{3\left(I_{3}=0\right) \text { cont }}<$ $A_{3 \max }\left(A_{3\left(I_{2}=0\right) \text { cont }}<A_{3 \max }\right)$. The dotted line in Figure 4 corresponds to $A_{3 \max }$.

Case (i): $n=3$ and $k=3$. Substitution of $n=3$ and $k=3$ into (13) leads to

$$
A_{3\left(I_{3}=0\right) \mathrm{cont}}=\frac{4 \sin ^{3} \tau_{p} \cos \tau_{p}}{6 \tau_{p}-\sin 6 \tau_{p}} .
$$

The solid line in Figure 4 corresponds to $A_{3\left(I_{3}=0\right) \text { cont }}$. It is easy to see that $\operatorname{sgn}\left[A_{3\left(I_{3}=0\right)}\right]=\operatorname{sgn}\left[\cos \tau_{p}\right]$ and $A_{3\left(I_{3}=0\right) \text { cont }}>$ $-1 / 3$. According to the above discussion concerning auxiliary waveform $g(\tau)$, waveform $i_{(k=3) \text { cont }}(\tau)$ with $A_{3}=A_{3\left(I_{3}=0\right) \text { cont }}$ is nonnegative if $A_{3\left(I_{3}=0\right) \text { cont }} \leq A_{3 \max }$. Substituting (25) and (22) into $A_{3\left(I_{3}=0\right) \text { cont }} \leq A_{3 \max }$ and solving the resulting relation for $\tau_{p}$, we obtain $2 \tau_{p} \geq 97.4^{\circ}$. Consequently, waveform of type $i_{(k=3) \text { cont }}(\tau)$ with $A_{3}=A_{3\left(I_{3}=0\right) \text { cont }}$ is nonnegative when $2 \tau_{p} \geq 97.4^{\circ}$ (second row in Table 1 ). 


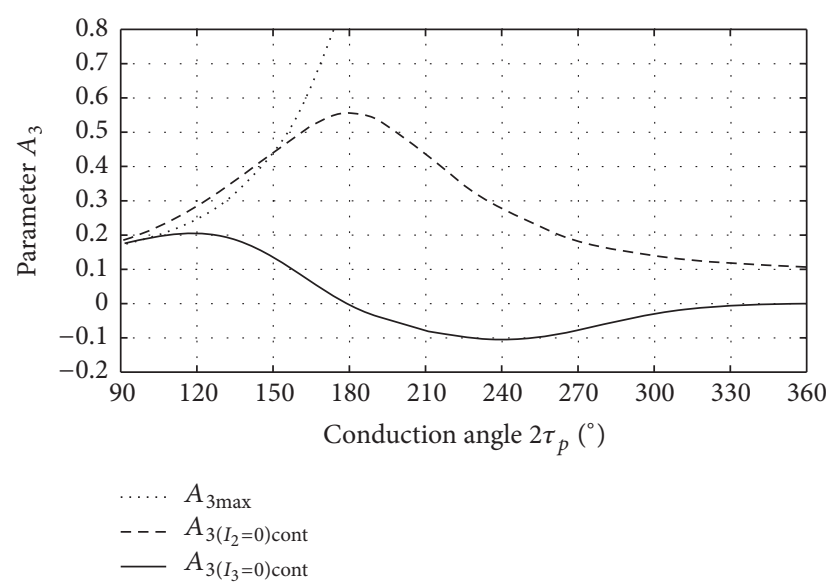

Figure 4: Parameter $A_{3}: A_{3 \max }$ (dotted line), $A_{3\left(I_{3}=0\right) \text { cont }}$ (solid line), and $A_{3\left(I_{2}=0\right) \text { cont }}$ (dashed line).

Case (iv): $n=2$ and $k=3$. For $n=2$ and $k=3$, expression (13) can be simplified to

$$
A_{3\left(I_{2}=0\right) \text { cont }}=\frac{5}{9\left(1+4 \cos ^{2} \tau_{p}\right)} .
$$

The dashed line in Figure 4 corresponds to $A_{3\left(I_{2}=0\right) \text { cont }}$. From (26), it immediately follows that $A_{3\left(I_{2}=0\right) \text { cont }}>1 / 9$. According to the above consideration involving auxiliary waveform $g(\tau)$, waveform $i_{(k=3) \text { cont }}(\tau)$ with $A_{3}=A_{3\left(I_{2}=0\right) \text { cont }}$ is nonnegative if $A_{3\left(I_{2}=0\right) \text { cont }} \leq A_{3 \max }$. Substituting (26) and (22) into $A_{3\left(I_{2}=0\right) \text { cont }} \leq A_{3 \max }$ and solving the resulting relation for $\tau_{p}$ yield $\tau_{p} \geq \arccos (1 / 4) \approx 75.52^{\circ}$. Consequently, waveform of type $i_{(k=3) \text { cont }}(\tau)$ with $A_{3}=A_{3\left(I_{2}=0\right) \text { cont }}$ is nonnegative when $2 \tau_{p} \geq 151.1^{\circ}$ (fifth row in Table 1 ).

Several examples of shapes of current waveforms of type $i_{(k=3) \text { cont }}(\tau)$ satisfying $I_{n(k=3)}=0$ are presented in Figure 5. Figure 5(a) corresponds to the case $n=3$ (case (i)), whereas Figure 5(b) corresponds to the case $n=2$ (case (iv)).

\section{Verification of the Analytical Results}

In this section, an independent numerical verification of the analytical results derived in Section 2 is presented. The values of the parameters of the optimal waveform of type $i_{\left(k, I_{n}=0\right)}(\tau), n \in\{2,3\}, k \in\{2,3\}$, obtained numerically are in full agreement with the values of the parameters calculated from the closed-form expressions derived in Section 2.

Algorithm 1 provides the procedure for numerical calculation of the parameters of the optimal current waveform of type $i_{\left(k, I_{n}=0\right)}(\tau)$ for prescribed conduction angle. Only conduction angles listed in Table 1 are considered, in accordance with the results of Section 2. The algorithm executes the brute force search for optimal current waveform through the set of nonnegative waveforms of type $i_{\left(k, I_{n}=0\right)}(\tau)$. Notice that, for the prescribed conduction angle, there exist only one continuous and an infinite number of discontinuous nonnegative waveforms of type $i_{\left(k, I_{n}=0\right)}(\tau)$.

A short description of Algorithm 1 is as follows: in line 2 , we initialize the value of counter $s$, and we choose step size $A_{0 \text {,step }}$ for $A_{0(k)} \in\left[A_{0, \text { min }}, A_{0, \text { max }}\right]$ and step size $\tau_{\text {step }}$ for $\tau \in\left[0, \tau_{p}\right]$ (because current waveform is an even function); in lines 3-5, we calculate $A_{k\left(I_{n}=0\right)}$ for each $A_{0(k)} \in$ $\left[A_{0, \text { min }}, A_{0, \text { max }}\right]$; in lines 6-11, we calculate $i_{\left(k, I_{n}=0\right)}(\tau)$, dc component, and amplitude of fundamental harmonic of $i_{\left(k, I_{n}=0\right)}(\tau)$ for each pair $\left(A_{0(k)}, A_{k\left(I_{n}=0\right)}\right)$; in line 12, we find the minimum value of $i_{\left(k, I_{n}=0\right)}(\tau)$ for each pair $\left(A_{0(k)}, A_{k\left(I_{n}=0\right)}\right)$; in lines 13-16, we calculate $\gamma_{I\left(k, I_{n}=0\right)}$ for each pair $\left(A_{0(k)}, A_{k\left(I_{n}=0\right)}\right)$ provided that corresponding $i_{\left(k, I_{n}=0\right)}(\tau)$ is nonnegative; in lines 17-24, we find $\gamma_{I\left(k, I_{n}=0\right)}^{*}$ as the maximum value of $\gamma_{I\left(k, I_{n}=0\right)}$ and corresponding pair $\left(A_{0(k)}^{*}, A_{k\left(I_{n}=0\right)}^{*}\right)$. Parameters of optimal waveform of type $i_{\left(k, I_{n}=0\right)}(\tau)$ are $A_{0(k)}^{*}, A_{k\left(I_{n}=0\right)}^{*}$, and $\gamma_{I\left(k, I_{n}=0\right)}^{*}$.

It is obvious that the numerical accuracy of the values of the parameters obtained by Algorithm 1 depends on chosen step size $\tau_{\text {step }}$ and step size $A_{0 \text {,step }}$. By numerical investigation, we find that $\tau_{\text {step }} \leq \tau_{p} / 1000$ and $A_{0 \text {,step }} \leq 0.001$ provide numerical stability of the three most significant decimal digits.

For example, for $n=3$ and $k=2$, numerical values of parameters $A_{2\left(I_{3}=0\right)}^{*}, A_{0(k=2)}^{*}$ and $\gamma_{I\left(k=2, I_{3}=0\right)}^{*}$ of optimal waveforms are calculated for $A_{0(k)} \in[-1,1.5], A_{0 \text {,step }}=$ $0.001,50^{\circ} \leq \tau_{p} \leq 180^{\circ}$, and $\tau_{\text {step }}=\tau_{p} / 1000$. On the other hand, values of parameters $A_{2\left(I_{3}=0\right) \text { cont }}, A_{0(k=2) \text { cont }}$, and $\gamma_{I\left(k=2, I_{3}=0\right) \text { cont }}$ are calculated from the closed-form expressions as follows:
(a) $A_{2\left(I_{3}=0\right) \text { cont }}$ from (17),
(b) $A_{0(k=2) \text { cont }}=-\cos \tau_{p}+A_{2\left(I_{3}=0\right) \operatorname{cont}} \cos 2 \tau_{p}$,
(c) substitution of $A_{0(k)}=A_{0(k=2) \text { cont }}$ and $A_{k\left(I_{n}=0\right)}=$ $A_{2\left(I_{3}=0\right) \text { cont }}$ into (9) yields $\gamma_{I\left(k=2, I_{3}=0\right) \text { cont }}$.

The values of parameters obtained by execution of Algorithm 1 and values calculated from the closed-form expressions are presented in Figure 6. Full agreement between the numerical and analytical results can be observed for all considered conduction angles.

For all remaining pairs $(n, k) \in\{(3,3),(2,2),(2,3)\}$, full agreement between analytical and numerical results is also confirmed (not shown here).

\section{Maximal Efficiency of Class-F and Inverse Class-F PA}

Maximal efficiencies of class-F PA and inverse class-F PA with current waveforms of type (1) are provided in Sections 4.1 and 4.2 , respectively. Our consideration in this section involves only conduction angles listed in Table 1.

Efficiency $\eta$ of PA can be expressed via basic waveform parameters $\gamma_{I}$ and $\gamma_{V}$ of current and voltage waveforms (see, e.g., [1]) as follows:

$$
\eta=\frac{\gamma_{I} \gamma_{V}}{2}
$$

As it has been shown in Section 2, optimal current waveform of type (1) for class-F (inverse class-F) PA is a continuous waveform $i_{(k) \text { cont }}(\tau)$ of type (6) satisfying condition $I_{3(k)}=0$ $\left(I_{2(k)}=0\right)$. 


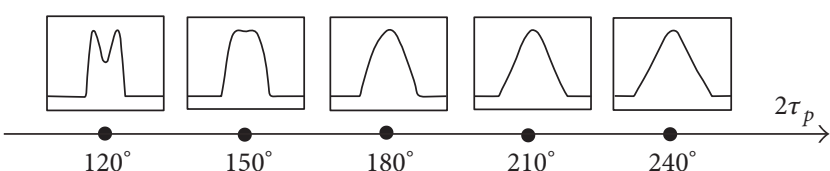

(a)

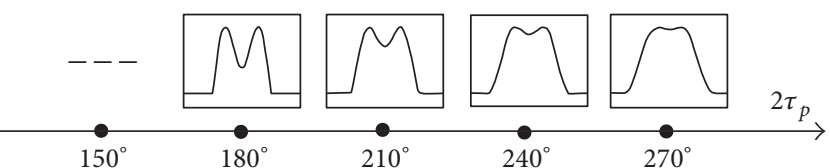

(b)

FIGURE 5: Shapes of current waveform of type $i_{(k=3) \text { cont }}(\tau)$ with (a) $A_{3}=A_{3\left(I_{3}=0\right) \text { cont }}$ and (b) $A_{3}=A_{3\left(I_{2}=0\right) \text { cont }}$.

Input:

$n, k / /(n, k) \in\{(3,3),(3,2),(2,2),(2,3)\}$

$I_{\mathrm{P}} / / I_{\mathrm{P}}>0$, e.g. $I_{\mathrm{P}}=1$ (the value of $I_{\mathrm{P}}$ has no influence on the output results)

$A_{0, \min }, A_{0, \max }, q_{\max } / / A_{0(k)}\left(1 \cdots q_{\max }\right) \in\left[A_{0, \min }, A_{0, \max }\right]$, e.g. $A_{0, \min }=-1, A_{0, \max }=1.5$

$\tau_{p} / /$ half of the conduction angle

$r_{\max } / / \tau\left(1 \cdots r_{\max }\right) \in\left[0, \tau_{p}\right]$ (current waveform is an even function of $\tau$ ) Output:

$\gamma_{I\left(k, I_{n}=0\right)}^{*}, A_{0(k)}^{*}, A_{k\left(I_{n}=0\right)}^{*} / /$ parameters of optimal waveform of type $i_{\left(k, I_{n}=0\right)}(\tau)$

(1) begin

(2) $s \leftarrow 0 ; A_{0, \text { step }} \leftarrow\left(A_{0, \max }-A_{0, \min }\right) /\left(q_{\max }-1\right) ; \tau_{\text {step }} \leftarrow \tau_{p} /\left(r_{\max }-1\right)$;

(3) for $q=1$ to $q_{\max }$ do

(4) $\quad A_{0(k)} \leftarrow A_{0, \min }+(q-1) A_{0, \text { step }}$;

(5) $\quad A_{k\left(I_{n}=0\right)} \leftarrow\left(\left(2 A_{0(k)} \operatorname{sinc} n \tau_{p}+\operatorname{sinc}(n-1) \tau_{p}+\operatorname{sinc}(n+1) \tau_{p}\right) /\left(\operatorname{sinc}(k-n) \tau_{p}+\operatorname{sinc}(k+n) \tau_{p}\right)\right) ; / /$ expression (8)

(6) $\quad I_{\mathrm{dc}} \leftarrow 0 ; I_{1} \leftarrow 0$;

(7) for $r=1$ to $r_{\max }$ do

(8) $\quad \tau \leftarrow(r-1) \tau_{\text {step; }}$;

(9) $\quad i_{\left(k, I_{n}=0\right)}(r) \leftarrow I_{\mathrm{P}}\left(A_{0(k)}+\cos \tau-A_{k\left(I_{n}=0\right)} \cos k \tau\right)$;

(10) $\quad I_{\mathrm{dc}} \leftarrow I_{\mathrm{dc}}+\left(\tau_{\text {step }} / \pi\right) i_{\left(k, I_{n}=0\right)}(r) ; I_{1} \leftarrow I_{1}+\left(2 \tau_{\text {step }} / \pi\right) i_{\left(k, I_{n}=0\right)}(r) \cos \tau$;

(11) end for // end $r$-loop

(12) $\quad i_{\text {min }} \leftarrow \min _{r} i_{\left(k, I_{n}=0\right)}\left(1 \cdots r_{\max }\right)$;

(13) if $i_{\min } \geq 0$ do

(16)

(17) $s_{\max } \leftarrow s$;

(18) if $s_{\max }=0$ do

(19) print "no solution found - increase the value of $A_{0, \max }$ and try again"

(20) else

(24) end if

$\gamma_{I\left(k, I_{n}=0\right)}^{*} \leftarrow \max _{s}\left(\gamma_{I\left(k, I_{n}=0\right)}\left(1 \cdots s_{\max }\right)\right) ;$

find $s^{*}$ such that $\gamma_{I\left(k, I_{n}=0\right)}\left(s^{*}\right)=\gamma_{I\left(k, I_{n}=0\right)}^{*}$;/ find index $s^{*}$ of the maximum element of $\gamma_{I\left(k, I_{n}=0\right)}$

(25) end.

Algorithm 1

For continuous waveforms of type (6), parameter $A_{0(k)}$ is given by (7). Substitution of (7) into (2)-(3) leads to the following expressions for Fourier coefficients of dc component and fundamental harmonic of waveform of type (6):

$$
\begin{gathered}
I_{\mathrm{dc}(k) \text { cont }}=\frac{I_{\mathrm{P}} \tau_{p}}{\pi}\left\{\operatorname{sinc} \tau_{p}-\cos \tau_{p}\right. \\
\left.-A_{k}\left[\operatorname{sinc} k \tau_{p}-\cos k \tau_{p}\right]\right\},
\end{gathered}
$$

$$
\begin{aligned}
& I_{1(k) \text { cont }}=\frac{I_{\mathrm{P}} \tau_{p}}{\pi}\left\{1-\operatorname{sinc} 2 \tau_{p}\right. \\
&\left.-k A_{k}\left[\operatorname{sinc}(k-1) \tau_{p}-\operatorname{sinc}(k+1) \tau_{p}\right]\right\} .
\end{aligned}
$$

Therefore, basic waveform parameter $\gamma_{I(k) \text { cont }}=I_{1(k) \text { cont }} /$ $I_{\mathrm{dc}(k) \text { cont }}$ of waveform $i_{(k) \text { cont }}(\tau)$ can be expressed as 


$$
\begin{aligned}
& \gamma_{I(k) \mathrm{cont}} \\
& =\frac{1-\operatorname{sinc} 2 \tau_{p}-k A_{k}\left[\operatorname{sinc}(k-1) \tau_{p}-\operatorname{sinc}(k+1) \tau_{p}\right]}{\operatorname{sinc} \tau_{p}-\cos \tau_{p}-A_{k}\left[\operatorname{sinc} k \tau_{p}-\cos k \tau_{p}\right]} .
\end{aligned}
$$

We denote basic waveform parameter of continuous waveform $i_{(k) \text { cont }}(\tau)$ with $A_{k}=A_{k\left(I_{n}=0\right) \text { cont }}$ by $\gamma_{I\left(k, I_{n}=0\right) \text { cont }}$. This parameter can be easily obtained from (30) by substituting $A_{k}=A_{k\left(I_{n}=0\right) \text { cont }}$,

$$
\begin{aligned}
& \gamma_{I\left(k, I_{n}=0\right) \text { cont }} \\
& =\frac{1-\operatorname{sinc} 2 \tau_{p}-k A_{k\left(I_{n}=0\right) \operatorname{cont}}\left[\operatorname{sinc}(k-1) \tau_{p}-\operatorname{sinc}(k+1) \tau_{p}\right]}{\operatorname{sinc} \tau_{p}-\cos \tau_{p}-A_{k\left(I_{n}=0\right) \operatorname{cont}}\left[\operatorname{sinc} k \tau_{p}-\cos k \tau_{p}\right]} .
\end{aligned}
$$

4.1. Maximal Efficiency of Class-F PA. In this subsection, we provide maximal efficiency of class-F PA with current waveform of type $i_{\left(k, I_{3}=0\right)}(\tau)$ for $k=2$ or $k=3$. In both cases, we assume that voltage waveform is an optimal waveform of type (4) with $n=3$ which reads (see, e.g., [1])

$$
v_{(n=3)}(\tau)=V_{\mathrm{dc}}\left[1-\frac{2}{\sqrt{3}} \cos \tau+\frac{\sqrt{3}}{9} \cos 3 \tau\right] \text {. }
$$

Basic waveform parameter $\gamma_{V}$ of $v_{(n=3)}(\tau)$ is $\gamma_{V(n=3)}=2 / \sqrt{3}$.

According to the results of Section 2, optimal current waveform, which in pair with voltage waveform (32) provides maximal efficiency of class-F PA, is a continuous waveform of type (6) with parameter $A_{k}=A_{k\left(I_{3}=0\right) \text { cont }}$. For $k=2$, parameter $A_{2\left(I_{3}=0\right) \text { cont }}$ is given by (17), whereas for $k=3$ parameter $A_{3\left(I_{3}=0\right) \text { cont }}$ is given by (25). Solid lines in Figures 2 and 4 correspond to parameters $A_{2\left(I_{3}=0\right) \text { cont }}$ and $A_{3\left(I_{3}=0\right) \text { cont }}$, respectively.

A number of shapes of optimal current waveforms of class-F PA are presented in Figures 3(a) and 5(a). Figure 5(a) corresponds to continuous waveform $i_{(k=3) \text { cont }}(\tau)$ with $A_{3}=$ $A_{3\left(I_{3}=0\right) \text { cont }}$ (case (i): $n=k=3$ ), whereas Figure 3(a) corresponds to continuous waveform $i_{(k=2) \text { cont }}(\tau)$ with $A_{2}=$ $A_{2\left(I_{3}=0\right) \text { cont }}$ (case (ii): $n=3$ and $k=2$ ).

According to (27), efficiency of class-F PA with voltage waveform (32) and current waveform of type $i_{(k) \text { cont }}(\tau)$ with $A_{k}=A_{k\left(I_{3}=0\right) \text { cont }}$ for $k \in\{2,3\}$ is equal to

$$
\eta_{\mathrm{F}(k)}=\frac{1}{\sqrt{3}} \gamma_{I\left(k, I_{3}=0\right) \mathrm{cont}} .
$$

Parameter $\gamma_{I\left(k=2, I_{3}=0\right) \text { cont }}$ can be obtained from (31) by substituting $n=3, k=2$, and $A_{2\left(I_{3}=0\right) \text { cont }}$ from (25). Parameter $\gamma_{I\left(k=3, I_{3}=0\right) \text { cont }}$ can be obtained from (31) by substituting $n=$ $3, k=3$, and $A_{3\left(I_{3}=0\right) \text { cont }}$ from (17). In Box 1, we provide a brief overview of the calculation of maximal efficiency of class-F PA.

Maximal efficiency of class-F PA is presented in Figure 7. The solid line corresponds to $k=3$ (case (i)), whereas the dashed line corresponds to $k=2$ (case (ii)). For prescribed $k$, maximal efficiency of class-F PA is a function of conduction angle only. The efficiency monotonically decreases with the increase of conduction angle as in classical modes of PA operation.

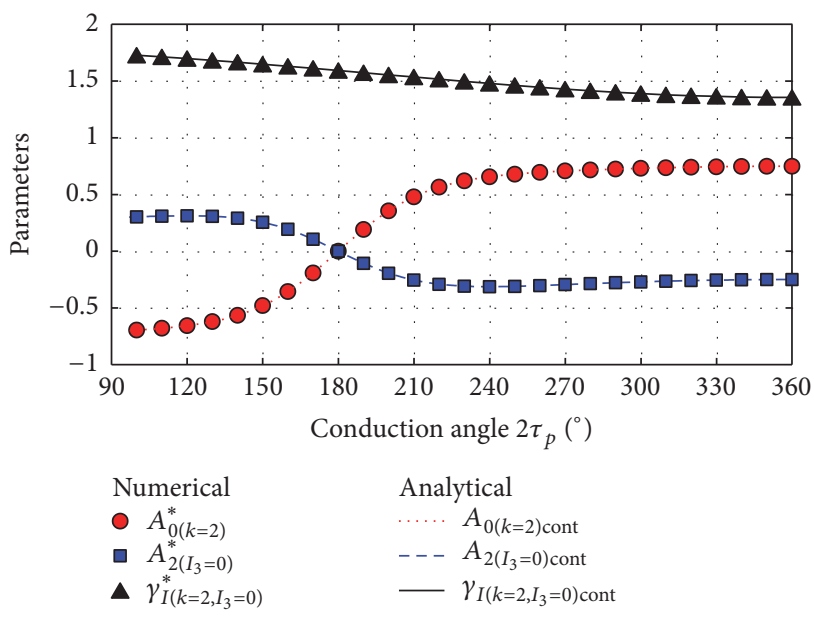

Figure 6: Parameters of optimal current waveform with $n=3$ and $k=2$. Numerical results: $A_{0(k=2)}^{*}$ (red circle dots), $A_{2\left(I_{3}=0\right)}^{*}$ (blue square dots), and $\gamma_{I\left(k=2, I_{3}=0\right)}^{*}$ (black triangle dots). Analytical results: $A_{0(k=2) \text { cont }}$ (red dotted line), $A_{2\left(I_{3}=0\right) \text { cont }}$ (blue dashed line), and $\gamma_{I\left(k=2, I_{3}=0\right) \text { cont }}$ (black solid line).

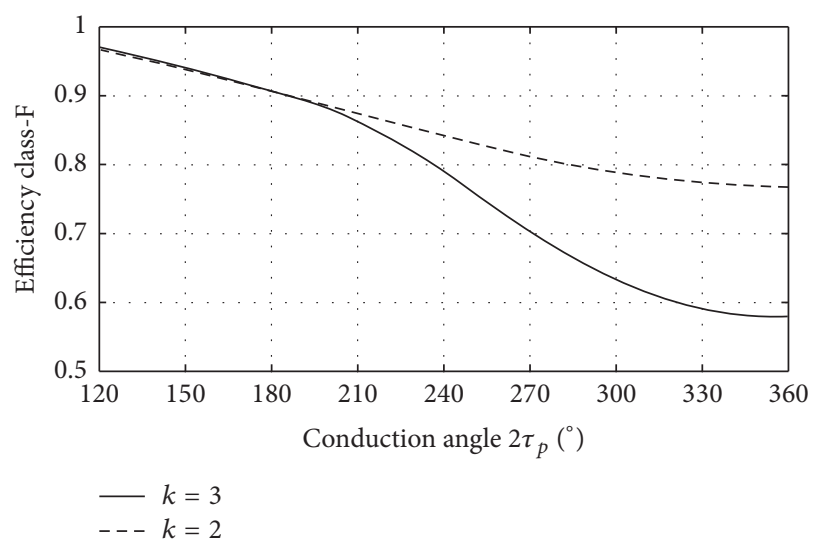

FIGURE 7: Maximal efficiency of class-F PA. Solid line corresponds to $k=3$ and dashed line corresponds to $k=2$.

For conduction angle $2 \tau_{p}=180^{\circ}$ (so-called class-B conduction angle), from Box 1 , we obtain $A_{2\left(I_{3}=0\right) \text { cont }}=0$ and $A_{3\left(I_{3}=0\right) \text { cont }}=0$. Thus, for $2 \tau_{p}=180^{\circ}$, optimal waveform is equal to $i_{\text {classic }}(\tau)$ (see (5)). Furthermore, we obtain $\eta_{\mathrm{F}}=$ $\sqrt{3} \pi / 6$, which is equal to the maximum efficiency $\eta=$ $(\pi / 6) \cot (\pi / 6)$ of class-F PA with voltage waveform (32) [10].

For conduction angle $2 \tau_{p}=126^{\circ}$ and $n=k=3$, from Box 1, we obtain $A_{3\left(I_{3}=0\right) \text { cont }}=0.2043$ and $\eta_{\mathrm{F}(k=3)}=0.9636$, which are in agreement with the results presented in [17] (see also [5]).

These examples also confirm our analytically obtained results presented in Section 2.

4.2. Maximal Efficiency of Inverse Class-F PA. In this subsection, we provide maximal efficiency of inverse class-F PA with current waveform of type $i_{\left(k, I_{2}=0\right)}(\tau)$ for $k=2$ or $k=3$. In 


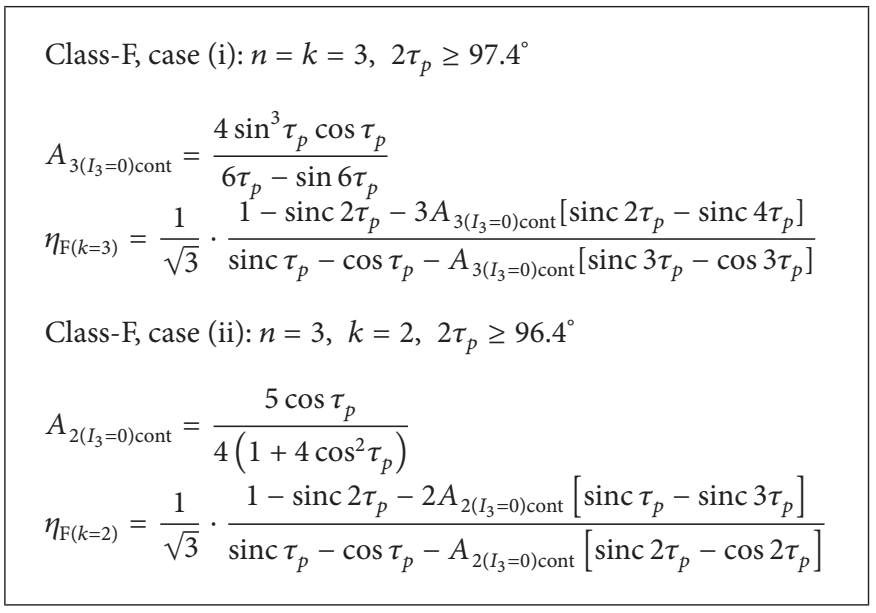

Box 1: Closed-form expressions used for the calculation of maximal efficiency of class-F PA.

both cases, we assume that voltage waveform is an optimal waveform of type (4) with $n=2$ which reads (see, e.g., [1])

$$
v_{(n=2)}(\tau)=V_{\mathrm{dc}}\left[1-\sqrt{2} \cos \tau+\frac{1}{2} \cos 2 \tau\right] .
$$

Basic waveform parameter of $v_{(n=2)}(\tau)$ is $\gamma_{V(n=2)}=\sqrt{2}$.

According to the results of Section 2, optimal current waveform, which in pair with voltage waveform (34) provides maximal efficiency of inverse class-F PA, is a continuous waveform of type $i_{(k) \text { cont }}(\tau)$ with parameter $A_{k}=A_{k\left(I_{2}=0\right) \text { cont }}$. For $k=2$, parameter $A_{2\left(I_{2}=0\right) \text { cont }}$ is given by (18), whereas for $k=3$ parameter $A_{3\left(I_{2}=0\right) \text { cont }}$ is given by (26). The dashed lines in Figures 2 and 4 correspond to parameters $A_{2\left(I_{2}=0\right) \text { cont }}$ and $A_{3\left(I_{2}=0\right) \text { cont }}$, respectively.

Several examples of shapes of current waveforms that provide maximal efficiency of inverse class-F PA are presented in Figures 3(b) and 5(b). Figure 3(b) corresponds to continuous waveform $i_{(k=2) \text { cont }}(\tau)$ with $A_{2}=A_{2\left(I_{2}=0\right) \text { cont }}$ (case (iii): $n=$ $k=2$ ), whereas Figure 5(b) corresponds to continuous waveform $i_{(k=3) \text { cont }}(\tau)$ with $A_{3}=A_{3\left(I_{2}=0\right) \text { cont }}$ (case (iv): $n=2$ and $k=3$ ).

According to (27), efficiency of inverse class-F PA with voltage waveform (34) and current waveform of type $i_{(k) \text { cont }}(\tau)$ with $A_{k}=A_{k\left(I_{2}=0\right) \text { cont }}$ for $k \in\{2,3\}$ is equal to

$$
\eta_{\mathrm{IF}(k)}=\frac{1}{\sqrt{2}} \gamma_{I\left(k, I_{2}=0\right) \mathrm{cont}} .
$$

Parameter $\gamma_{I\left(k=2, I_{2}=0\right) \text { cont }}$ can be obtained from (31) by substi-

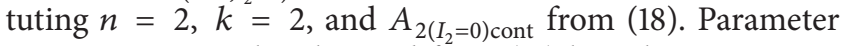
$\gamma_{I\left(k=3, I_{2}=0\right) \text { cont }}$ can be obtained from (31) by substituting $n=$ $2, k=3$, and $A_{3\left(I_{2}=0\right) \text { cont }}$ from (26). In Box 2, we provide a brief overview of the calculation of maximal efficiency of inverse class-F PA.

Maximal efficiency of inverse class-F PA is presented in Figure 8. The solid line corresponds to $k=2$ (case (iii)), whereas the dashed line corresponds to $k=3$ (case (iv)). For prescribed $k$, maximal efficiency of inverse classF PA is a function of conduction angle only. The efficiency

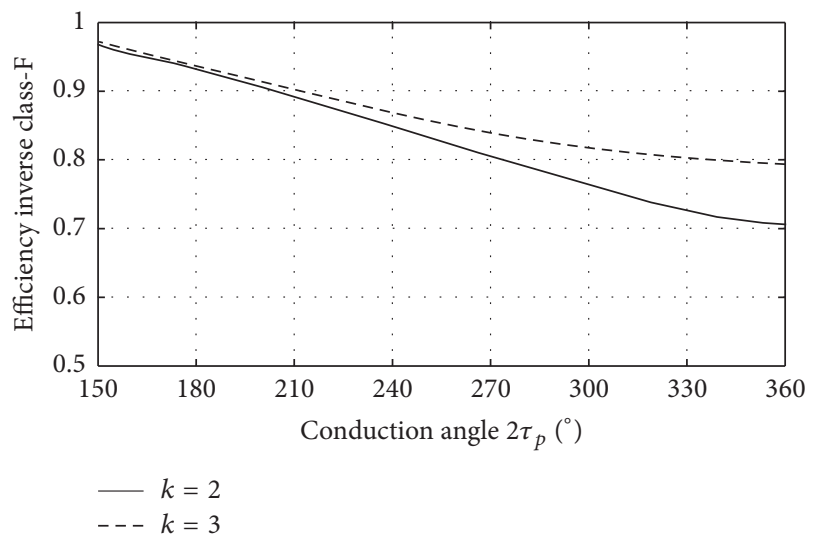

Figure 8: Maximal efficiency of inverse class-F PA. Solid line corresponds to $k=2$ and dashed line corresponds to $k=3$.

monotonically decreases with the increase of conduction angle as in classical modes of PA operation.

For conduction angle $2 \tau_{p}=160^{\circ}$ and $k=n=2$, from Box 2, we obtain $A_{2\left(I_{2}=0\right)}=0.4090$ and $\eta_{\mathrm{IF}(k=2)}=0.9560$, which are in agreement with the results presented in [17] (see also [5]).

Also, for conduction angle $2 \tau_{p}=246^{\circ}$ and $k=n=2$, we obtain $A_{2\left(I_{2}=0\right)}=0.2006$ and $\eta_{\mathrm{IF}(k=2)}=0.8407$, which are in agreement with the results presented in [18].

These two examples provide another verification of analytical results presented in Section 2.

\section{Suboptimal Continuous Current Waveforms in PA Efficiency Analysis}

In this section, we consider continuous current waveforms of type $(6), k \in\{2,3\}$, with second $I_{2(k) \text { cont }}$ or third $I_{3(k) \text { cont }}$ harmonic Fourier coefficient being nonpositive (in contrast to the previous more strict condition that either coefficient of second or third harmonic is equal to zero). Thus, here we extend our analysis and consider suboptimal 
Inverse class-F, case (iii), $n=k=2,2 \tau_{p} \geq 146.4^{\circ}$

$$
\begin{aligned}
& A_{2\left(I_{2}=0\right) \text { cont }}=\frac{8 \sin ^{3} \tau_{p}}{3\left(4 \tau_{p}-\sin 4 \tau_{p}\right)} \\
& \eta_{\operatorname{IF}(k=2)}=\frac{1}{\sqrt{2}} \cdot \frac{1-\operatorname{sinc} 2 \tau_{p}-2 A_{2\left(I_{2}=0\right) \operatorname{cont}}\left[\operatorname{sinc} \tau_{p}-\operatorname{sinc} 3 \tau_{p}\right]}{\operatorname{sinc} \tau_{p}-\cos \tau_{p}-A_{2\left(I_{2}=0\right) \operatorname{cont}}\left[\operatorname{sinc} 2 \tau_{p}-\cos 2 \tau_{p}\right]} \\
& \text { Inverse class-F, case (iv), } n=2, k=3,2 \tau_{p} \geq 151.1^{\circ} \\
& A_{3\left(I_{2}=0\right) \text { cont }}=\frac{5}{9\left(1+4 \cos ^{2} \tau_{p}\right)} \\
& \eta_{\operatorname{IF}(k=3)}=\frac{1}{\sqrt{2}} \cdot \frac{1-\operatorname{sinc} 2 \tau_{p}-3 A_{3\left(I_{2}=0\right) \operatorname{cont}}\left[\operatorname{sinc} 2 \tau_{p}-\operatorname{sinc} 4 \tau_{p}\right]}{\operatorname{sinc} \tau_{p}-\cos \tau_{p}-A_{3\left(I_{2}=0\right) \operatorname{cont}}\left[\operatorname{sinc} 3 \tau_{p}-\cos 3 \tau_{p}\right]}
\end{aligned}
$$

Box 2: Closed-form expressions used for the calculation of maximal efficiency of inverse class-F PA.

continuous current waveforms, which are also of interest for the modeling of waveforms for PA design. Moreover, we provide two case studies involving continuous current waveforms with $k=2$ satisfying $I_{3(k=2) \text { cont }}<0$ (case study 1 ) or $I_{2(k=2) \text { cont }}<0$ (case study 2 ) in PA efficiency analysis.

\subsection{Continuous Current Waveforms with Nonpositive Fourier} Coefficient of the Second (Third) Harmonic. Fourier coefficient of the $n$th harmonic of current waveform of type (6) can be obtained by substituting (7) into (3):

$$
\begin{gathered}
I_{n(k) \mathrm{cont}}=\frac{I_{\mathrm{P}} \tau_{p}}{n \pi}\left\{\operatorname{sinc}(n-1) \tau_{p}-\operatorname{sinc}(n+1) \tau_{p}\right. \\
\left.-k A_{k}\left[\operatorname{sinc}(k-n) \tau_{p}-\operatorname{sinc}(k+n) \tau_{p}\right]\right\} .
\end{gathered}
$$

It is easy to show that $\operatorname{sinc}(k-n) \tau_{p}-\operatorname{sinc}(k+n) \tau_{p}>0$, for $2 \tau_{p} \in\left(0,360^{\circ}\right), k \in\{2,3\}$, and $n \in\{2,3\}$. Consequently, Fourier coefficient of the $n$th harmonic is nonpositive when

$$
A_{k} \geq \frac{\operatorname{sinc}(n-1) \tau_{p}-\operatorname{sinc}(n+1) \tau_{p}}{k\left[\operatorname{sinc}(k-n) \tau_{p}-\operatorname{sinc}(k+n) \tau_{p}\right]} .
$$

The value of $A_{k}$ for which (37) becomes equality is $A_{k\left(I_{n}=0\right) \text { cont }}$ (see (13)). Thus, (37) can be rewritten as

$$
A_{k} \geq A_{k\left(I_{n}=0\right) \text { cont }}
$$

As it has been shown in Section 2, expression for $A_{k\left(I_{n}=0\right) \text { cont }}$ can be reduced to:

(i) expression (25) for $(n, k)=(3,3)$; parameter $A_{3\left(I_{3}=0\right) \text { cont }}$ is plotted with solid line in Figure 4,

(ii) expression (17) for $(n, k)=(3,2)$; parameter $A_{2\left(I_{3}=0\right) \text { cont }}$ is plotted with solid line in Figure 2,

(iii) expression (18) for $(n, k)=(2,2)$; parameter $A_{2\left(I_{2}=0\right) \text { cont }}$ is plotted with dashed line in Figure 2,

(iv) expression (26) for $(n, k)=(2,3)$; parameter $A_{3\left(I_{2}=0\right) \text { cont }}$ is plotted with dashed line in Figure 4.
Coefficients of fundamental and third harmonic $(n=3)$ of optimal voltage waveform (32) for class-F PA have opposite signs. Similarly, coefficients of fundamental and second harmonic ( $n=2$ ) of optimal voltage waveform (34) for inverse class-F PA have opposite signs. Therefore, when the output network of PA is passive, voltage waveform (32) can be paired with current waveform of type (6), provided that the third-harmonic Fourier coefficient of current waveform is nonpositive. Analogously, voltage waveform (34) can be paired with current waveform of type (6) provided that the second-harmonic Fourier coefficient of current waveform is nonpositive.

For example, substitution of $n=3, k=2$, and $2 \tau_{p}=$ $180^{\circ}$ (class-B conduction angle) into (37) yields $A_{2} \geq 0$. According to the above discussion, Fourier coefficient of the third harmonic is positive for $A_{2}<0$, equal to zero for $A_{2}=A_{2\left(I_{3}=0\right) \text { cont }}=0$, and negative for $A_{2}>0$. Therefore, small changes of parameter $A_{2}$ around the optimal value $A_{2\left(I_{3}=0\right) \text { cont }}=0$ may cause sign changes of the third-harmonic current component. When output network of PA is passive, sign change of the third-harmonic current component causes sign change of the third-harmonic voltage component, which eventually may lead to a significant decrease of efficiency of class-F PA.

Similarly, substitution of $n=k=3$ and $2 \tau_{p}=180^{\circ}$ into (37) yields $A_{3} \geq 0$. Fourier coefficient of the third harmonic of continuous current waveform (6) is nonpositive for all $A_{3} \geq 0$ and positive for all $A_{3}<0$. Therefore, small change of parameter $A_{3}$ from the optimal value $A_{3\left(I_{3}=0\right) \text { cont }}=0$ may also lead to a significant decrease of efficiency of class-F PA.

5.2. Case Studies. Here, we consider efficiency of PAs with suboptimal current waveforms of type (6) for $k=2$. We illustrate that with near-optimal current waveform almost maximal efficiency of class-F (inverse class-F) PA can be attained. In case study 1, the third-harmonic Fourier coefficient of current waveform is negative, whereas the second-harmonic Fourier coefficient is negative in case study 2. Corresponding voltages are also suboptimal waveforms: nonnegative 
waveform with dc component and fundamental and third harmonic in case study 1 and nonnegative waveform with $\mathrm{dc}$ component and fundamental and second harmonic in case study 2 .

Let us consider generic PA circuit shown in Figure 9. We assume that voltage and current waveforms at the output port of transistor are

$$
\begin{aligned}
& v(\tau)=1+a_{1 v} \cos \tau+b_{1 v} \sin \tau+a_{n v} \cos n \tau \\
& +b_{n v} \sin n \tau \text {, } \\
& i(\tau)=1+a_{1 i} \cos \tau+\sum_{m=2}^{\infty} a_{m i} \cos m \tau .
\end{aligned}
$$

Both waveforms are normalized such that $V_{\mathrm{dc}}=1$ and $I_{\mathrm{dc}}=1$. Under common assumptions that $C_{b}$ behaves as short circuit and $L_{\mathrm{ch}}$ behaves as open circuit at fundamental and higher harmonics, voltage and current waveforms at the load are

$$
\begin{aligned}
& v_{L}(\tau)=a_{1 v} \cos \tau+b_{1 v} \sin \tau+a_{n v} \cos n \tau+b_{n v} \sin n \tau \\
& i_{L}(\tau)=-a_{1 i} \cos \tau-\sum_{m=2}^{\infty} a_{m i} \cos m \tau
\end{aligned}
$$

The load impedance is equal to $\underline{z}_{1}=-\left(a_{1 v}-\mathrm{j} b_{1 v}\right) / a_{1 i}$ at fundamental harmonic, to $\underline{z}_{n}=-\left(a_{n v}-\mathrm{j} b_{n v}\right) / a_{n i}$ at $n$th harmonic, and to $\underline{z}_{m}=0$ at $m$ th harmonic, $m>1$ and $m \neq n$. When load is passive $\left(\operatorname{Re}\left\{\underline{z}_{1}\right\} \geq 0\right.$ and $\left.\operatorname{Re}\left\{\underline{z}_{n}\right\} \geq 0\right)$, products $a_{1 v} a_{1 i}$ and $a_{n v} a_{n i}$ are nonpositive.
Efficiency of PA with normalized waveforms (39) can be calculated as (e.g., see, [14])

$$
\eta=-\frac{a_{1 i} a_{1 v}}{2}
$$

Case Study 1. In this case study, we analyze the efficiency of PA with current waveform of type (6) for $k=2$ and negative Fourier coefficient of the third harmonic. This type of current waveform can be considered as suboptimal current waveform of class-F PA. Corresponding voltage waveform of $\mathrm{PA}$ is suboptimal nonnegative waveform with dc component and fundamental and third harmonic.

Let us introduce two current waveforms, one of them being very close to the optimal for class-F PA. More precisely, these waveforms are normalized waveforms of type (6) with

$$
\begin{aligned}
& \text { subcase }(1 \mathrm{a}): I_{\mathrm{dc}(k=2) \text { cont }}=1, k=2,2 \tau_{p}=160^{\circ} \text {, and } \\
& A_{2}=0.3, \\
& \text { subcase }(1 \mathrm{~b}): I_{\mathrm{dc}(k=2) \text { cont }}=1, k=2,2 \tau_{p}=160^{\circ} \text {, and } \\
& A_{2}=0.2 .
\end{aligned}
$$

According to (17), optimal value of parameter $A_{2}$ for class-F $\mathrm{PA}$ is $A_{2\left(I_{3}=0\right) \text { cont }}=0.1937$. In both subcases, $A_{2}>A_{2\left(I_{3}=0\right) \text { cont }}$ and therefore the third-harmonic Fourier coefficients of both waveforms are negative. The value of $A_{2}$ in subcase (1b) is very close to the optimal.

In subcase (1a), substitution of $I_{\mathrm{dc}(k=2) \text { cont }}=1,2 \tau_{p}=$ $160^{\circ}, k=2$, and $A_{2}=0.3$ into (28) leads to $I_{\mathrm{P}}=10.56$. Furthermore, from (6), we obtain normalized form of current waveform

$$
i^{(1 \mathrm{a})}(\tau)= \begin{cases}10.56\left[\cos \tau-\cos 80^{\circ}-0.3\left(\cos 2 \tau-\cos 160^{\circ}\right)\right], & |\tau| \leq 80^{\circ}, \\ 0, & 80^{\circ} \leq|\tau| \leq 180^{\circ}\end{cases}
$$

Fourier coefficients of fundamental and third harmonic of $i^{(1 \mathrm{a})}(\tau)$ are

$$
\begin{aligned}
& a_{1 i}^{(1 \mathrm{a})}=1.5505, \\
& a_{3 i}^{(1 \mathrm{a})}=-0.2040 .
\end{aligned}
$$

In subcase $(1 \mathrm{~b})$, substitution of $I_{\mathrm{dc}(k=2) \text { cont }}=1,2 \tau_{p}=$ $160^{\circ}, k=2$, and $A_{2}=0.2$ into (28) leads to $I_{\mathrm{P}}=7.048$. From (6), we obtain normalized current waveform

$$
i^{(1 \mathrm{~b})}(\tau)= \begin{cases}7.048\left[\cos \tau-\cos 80^{\circ}-0.2\left(\cos 2 \tau-\cos 160^{\circ}\right)\right], & |\tau| \leq 80^{\circ}, \\ 0, & 80^{\circ} \leq|\tau| \leq 180^{\circ}\end{cases}
$$

Fourier coefficients of fundamental and third harmonic of $i^{(1 \mathrm{~b})}(\tau)$ are

$$
\begin{aligned}
& a_{1 i}^{(1 \mathrm{~b})}=1.6060, \\
& a_{3 i}^{(1 \mathrm{~b})}=-0.0081 .
\end{aligned}
$$

In both subcases, we assume that voltage is nonnegative waveform of type

$$
\begin{aligned}
v(\tau)= & 1+a_{1 v} \cos \tau+b_{1 v} \sin \tau+a_{3 v} \cos 3 \tau \\
& +b_{3 v} \sin 3 \tau .
\end{aligned}
$$


// input: $a_{1 \mathrm{i}}$ and $a_{3 \mathrm{i}}$;

(i) choose $\underline{z}_{3}=r_{3}+\mathrm{j} x_{3}$ such that $\left|\underline{z}_{3}\right| \leq 1 /\left|a_{3 i}\right|$ and $r_{3} \geq 0$;

(ii) calculate $a_{3 v}-\mathrm{j} b_{3 v}=-\underline{z}_{3} a_{3 i}$ and $\lambda_{3 v}=\sqrt{a_{3 v}^{2}+b_{3 v}^{2}}$;

(iii) if $27 \lambda_{3 v}^{2} \leq\left(1-2 a_{3 v}\right)^{3}$ then calculate $a_{1 v}=-1-a_{3 v}$ and $b_{1 v}=-3 b_{3 v}$;

else, calculate $\lambda_{1 v}=3\left(\sqrt[3]{\lambda_{3 v}}-\lambda_{3 v}\right), \varphi_{1 v}=(1 / 3) \operatorname{atan} 2\left(b_{3 v}, a_{3 v}\right), a_{1 v}=-\lambda_{1 v} \cos \varphi_{1 v}$ and $b_{1 v}=-\lambda_{1 v} \sin \varphi_{1 v}$;

(iv) calculate efficiency $\eta=-a_{1 i} a_{1 v} / 2$;

(v) calculate $\underline{z}_{1}=-\left(a_{1 v}-\mathrm{j} b_{1 v}\right) / a_{1 i}$ and $\underline{z}_{3 n}=\underline{z}_{3} / \operatorname{Re}\left\{\underline{z}_{1}\right\}$.

Algorithm 2

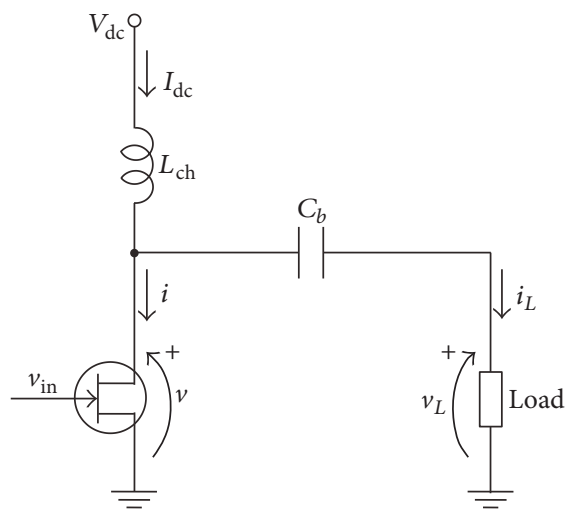

Figure 9: Generic PA circuit diagram.

For passive load, $\operatorname{Re}\left\{\underline{z}_{1}\right\} \geq 0$ along with $a_{1 i}^{(1 \mathrm{a})}>0$ (i.e., $a_{1 i}^{(1 \mathrm{~b})}>0$ ) implies $a_{1 v} \leq 0$. Also, $\operatorname{Re}\left\{\underline{z}_{3}\right\} \geq 0$ along with $a_{3 i}^{(1 \mathrm{a})}<0$ (i.e., $a_{3 i}^{(1 \mathrm{~b})}<0$ ) implies $a_{3 v} \geq 0$. In [14], it is proved that relation $\lambda_{3 v} \leq 1$ holds $\left(\lambda_{3 v}=\sqrt{a_{3 v}^{2}+b_{3 v}^{2}}\right)$ for all nonnegative waveforms of type (46). In subcase (1a), relation $\lambda_{3 v} \leq 1$ together with $\lambda_{3 v}=\left|\underline{z}_{3}\right|\left|a_{3 i}^{(1 \mathrm{a})}\right|$ implies $\left|\underline{z}_{3}\right| \leq 1 /\left|a_{3 i}^{(1 \mathrm{a})}\right|$. Therefore, in subcase (1a), values of impedance at the third harmonic are bounded by $\left|\underline{z}_{3}\right| \leq 1 /\left|a_{3 i}^{(1 \mathrm{a})}\right|$ and $\operatorname{Re}\left\{\underline{z}_{3}\right\} \geq 0$. Similarly, in subcase (1b), values of impedance at the third harmonic are bounded by $\left|\underline{z}_{3}\right| \leq 1 /\left|a_{3 i}^{(1 \mathrm{~b})}\right|$ and $\operatorname{Re}\left\{\underline{z}_{3}\right\} \geq 0$.

In what follows, we consider maximal efficiency of PA with current waveform (42) (i.e., (44)) and voltage waveform (46) provided that $\left|\underline{z}_{3}\right| \leq 1 /\left|a_{3 i}^{(1 \mathrm{a})}\right|$ and $\operatorname{Re}\left\{\underline{z}_{3}\right\} \geq 0$ in subcase (1a) (i.e., $\left|\underline{z}_{3}\right| \leq 1 /\left|a_{3 i}^{(1 \mathrm{~b})}\right|$ and $\operatorname{Re}\left\{\underline{z}_{3}\right\} \geq 0$ in subcase (1b)). The problem of finding maximal efficiency of PA with waveform pair (46) and (42) (i.e., (44)) for the prescribed third-harmonic impedance can be reduced to the problem of finding voltage waveform of type (46) with maximal absolute value of $a_{1 v}$ for prescribed coefficients of the third harmonic [14]. Fourier series expansion of (42) (i.e., (44)) contains only cosine terms and therefore the procedure for calculation of maximal efficiency of PA described by Algorithm 33 in [14] can be used in both subcases. For the sake of completeness, here we provide Algorithm 2, which is analogous to Algorithm 33 in [14]. To improve readability of the algorithm, we change the notation: $\theta_{0 v}-\xi_{v} / 3$ in [14] is replaced with $\varphi_{1 v}$.
Function $\operatorname{atan} 2(y, x)$, which appears in step (iii) of Algorithm 2, is defined as

$$
\operatorname{atan} 2(y, x)= \begin{cases}\arctan \left(\frac{y}{x}\right) & \text { if } x \geq 0, \\ \arctan \left(\frac{y}{x}\right)+\pi & \text { if } x<0, y \geq 0, \\ \arctan \left(\frac{y}{x}\right)-\pi & \text { if } x<0, y<0 .\end{cases}
$$

In subcase (1a), input of Algorithm 2 (coefficients $a_{1 i}$ and $a_{3 i}$ ) is given by (43). Maximal efficiency of PA with waveform pair (42) and (46), as a function of normalized third-harmonic impedance $\underline{z}_{3 n}=\underline{z}_{3} / \operatorname{Re}\left\{\underline{z}_{1}\right\}$, is presented in Figure $10(\mathrm{a})$. Efficiency of 0.89 is attained in the vicinity of $\underline{z}_{3 n}=1.26$ (corresponding to $\underline{z}_{3}=0.94$ ).

In subcase (1b), input of Algorithm 2 (coefficients $a_{1 i}$ and $a_{3 i}$ ) is given by (45). Maximal efficiency of PA with waveform pair (44) and (46), as a function of normalized third-harmonic impedance, is presented in Figure 10(b). Efficiency of 0.92 is achieved in the vicinity of $\underline{z}_{3 n}=33.16$ (corresponding to $\underline{z}_{3}=23.84$ ).

As we mentioned earlier, the optimal value of parameter $A_{2}$ is $A_{2\left(I_{3}=0\right) \text { cont }}=0.1937$. Corresponding maximal efficiency of class-F PA (see Box 1 ) is $\eta_{\mathrm{F}(k=2)}=0.9286$. Current waveform $i^{(1 \mathrm{~b})}(\tau)$ in subcase (1b) is very close to optimal current waveform for class-F PA and corresponding peak efficiency of 0.92 is very close to $\eta_{\mathrm{F}(k=2)}=0.9286$.

Case Study 2. In this case study, we analyze the efficiency of PA with current waveforms of type (6) for $k=2$ and negative Fourier coefficient of the second harmonic. This type of current waveform can be considered as suboptimal current waveform of inverse class-F PA. Corresponding voltage of PA is a suboptimal nonnegative waveform with dc component and fundamental and second harmonic.

Let us introduce two current waveforms, one of them being close to the optimal for inverse class-F PA. More precisely, these waveforms are normalized waveforms of type (6) with

subcase (2a): $I_{\mathrm{dc}(k=2) \text { cont }}=1, k=2,2 \tau_{p}=220^{\circ}$, and $A_{2}=0.4$,

subcase (2b): $I_{\mathrm{dc}(k=2) \text { cont }}=1, k=2,2 \tau_{p}=220^{\circ}$, and $A_{2}=0.34$. 


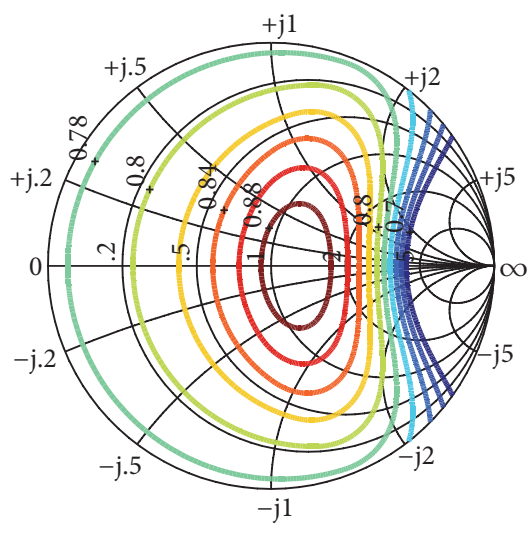

(a)

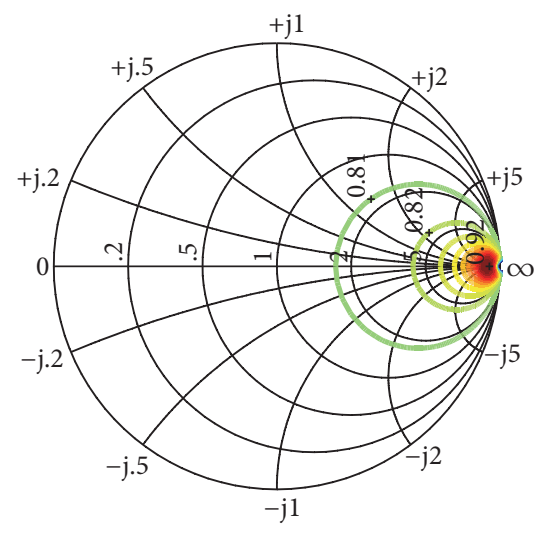

(b)

FIGURE 10: Maximal efficiency of PA with (a) current (42) and voltage (46) and (b) current (44) and voltage (46) as a function of normalized third-harmonic impedance.

According to (18), the optimal value of parameter $A_{2}$ for inverse class-F PA is $A_{2\left(I_{2}=0\right) \text { cont }}=0.3305$. In both subcases, $A_{2}>A_{2\left(I_{2}=0\right) \text { cont }}$ and therefore second-harmonic Fourier coefficients of both waveforms are negative. The value of $A_{2}$ in subcase (2b) is close to the optimal.
In subcase (2a), substitution of $I_{\mathrm{dc}(k=2) \text { cont }}=1,2 \tau_{p}=$ $220^{\circ}, k=2$, and $A_{2}=0.4$ into (28) leads to $I_{\mathrm{P}}=2.764$. Furthermore, from (6), we obtain normalized form of current waveform

$$
i^{(2 \mathrm{a})}(\tau)= \begin{cases}2.764\left[\cos \tau-\cos 110^{\circ}-0.4\left(\cos 2 \tau-\cos 220^{\circ}\right)\right], & |\tau| \leq 110^{\circ}, \\ 0, & 110^{\circ} \leq|\tau| \leq 180^{\circ} .\end{cases}
$$

Fourier coefficients of fundamental and second harmonic of $i^{(2 \mathrm{a})}(\tau)$ are

$$
\begin{aligned}
& a_{1 i}^{(2 \mathrm{a})}=1.1932, \\
& a_{2 i}^{(2 \mathrm{a})}=-0.1023 .
\end{aligned}
$$

In subcase (2b), substitution of $I_{\mathrm{dc}(k=2) \text { cont }}=1,2 \tau_{p}=$ $220^{\circ}, k=2$, and $A_{2}=0.34$ into (28) leads to $I_{\mathrm{P}}=2.606$. From (6), we obtain normalized current waveform

$$
i^{(2 \mathrm{~b})}(\tau)= \begin{cases}2.606\left[\cos \tau-\cos 110^{\circ}-0.34\left(\cos 2 \tau-\cos 220^{\circ}\right)\right], & |\tau| \leq 110^{\circ}, \\ 0, & 110^{\circ} \leq|\tau| \leq 180^{\circ} .\end{cases}
$$

Fourier coefficients of fundamental and second harmonic of $i^{(2 \mathrm{~b})}(\tau)$ are

$$
\begin{aligned}
& a_{1 i}^{(2 \mathrm{~b})}=1.2351, \\
& a_{2 i}^{(2 \mathrm{~b})}=-0.0132 .
\end{aligned}
$$

In both subcases, we assume that voltage is a nonnegative waveform of type

$$
\begin{aligned}
v(\tau)= & 1+a_{1 v} \cos \tau+b_{1 v} \sin \tau+a_{2 v} \cos 2 \tau \\
& +b_{2 v} \sin 2 \tau .
\end{aligned}
$$

For passive load, $\operatorname{Re}\left\{\underline{z}_{1}\right\} \geq 0$ along with $a_{1 i}^{(2 \mathrm{a})}>0$ (i.e., $a_{1 i}^{(2 \mathrm{~b})}>0$ ) implies $a_{1 v} \leq 0$. Also, $\operatorname{Re}\left\{\underline{z}_{2}\right\} \geq 0$ along with $a_{2 i}^{(2 \mathrm{a})}<0$ (i.e., $a_{2 i}^{(2 \mathrm{~b})}<0$ ) implies $a_{2 v} \geq 0$. In [14], it is proved that relation $\lambda_{2 v} \leq 1$ holds $\left(\lambda_{2 v}=\sqrt{a_{2 v}^{2}+b_{2 v}^{2}}\right)$ for all nonnegative waveforms of type (52). In subcase (2a), relations $\lambda_{2 v} \leq 1$ and $\lambda_{2 v}=\left|\underline{z}_{2}\right|\left|a_{2 i}^{(2 \mathrm{a})}\right|$ imply $\left|\underline{z}_{2}\right| \leq 1 /\left|a_{2 i}^{(2 \mathrm{a})}\right|$. Therefore, in subcase (2a), values of impedance at the second harmonic are bounded by $\left|\underline{z}_{2}\right| \leq 1 /\left|a_{2 i}^{(2 \mathrm{a})}\right|$ and $\operatorname{Re}\left\{\underline{z}_{2}\right\} \geq 0$. Similarly, in subcase (2b), values of impedance at the second harmonic are bounded by $\left|\underline{z}_{2}\right| \leq 1 /\left|a_{2 i}^{(2 b)}\right|$ and $\operatorname{Re}\left\{\underline{z}_{2}\right\} \geq 0$. 
// input: $a_{1 i}$ and $a_{2 i}$;

(i) choose $\underline{z}_{2}=r_{2}+\mathrm{j} x_{2}$ such that $\left|\underline{z}_{2}\right| \leq 1 /\left|a_{2 i}\right|$ and $r_{2} \geq 0$;

(ii) calculate $a_{2 v}-\mathrm{j} b_{2 v}=-\underline{z}_{2} a_{2 i}$ and $\lambda_{2 v}=\sqrt{a_{2 v}^{2}+b_{2 v}^{2}}$;

(iii) if $2 \lambda_{2 v} \leq 1-a_{2 v}$ then calculate $a_{1 v}=-1-a_{2 v}$ and $b_{1 v}=-2 b_{2 v}$;

else, calculate $\lambda_{1 v}=\sqrt{8 \lambda_{2 v}\left(1-\lambda_{2 v}\right)}, \varphi_{1 v}=(1 / 2) \operatorname{atan} 2\left(b_{2 v}, a_{2 v}\right), a_{1 v}=-\lambda_{1 v} \cos \varphi_{1 v}$ and $b_{1 v}=-\lambda_{1 v} \sin \varphi_{1 v}$;

(iv) calculate efficiency $\eta=-a_{1 i} a_{1 v} / 2$;

(v) calculate $\underline{z}_{1}=-\left(a_{1 v}-j b_{1 v}\right) / a_{1 i}$ and $\underline{z}_{2 n}=\underline{z}_{2} / \operatorname{Re}\left\{\underline{z}_{1}\right\}$.

Algorithm 3

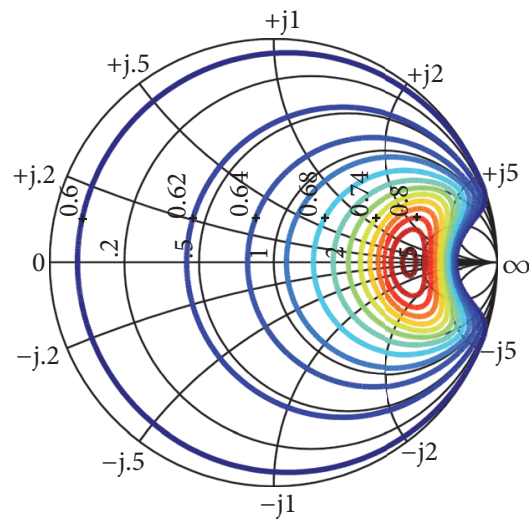

(a)

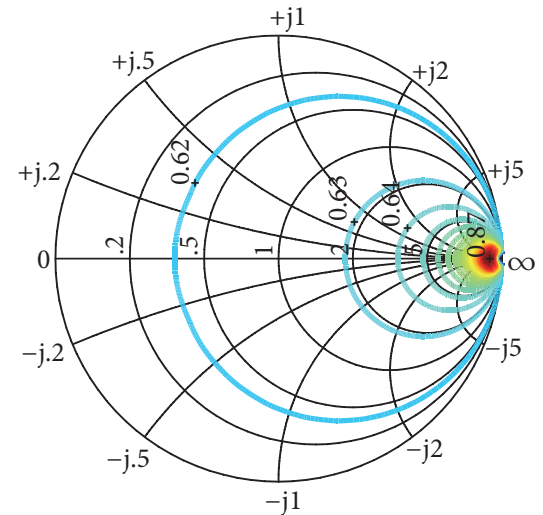

(b)

FIGURE 11: Maximal efficiency of PA with (a) current (48) and voltage (52) and (b) current (50) and voltage (52) as a function of normalized second-harmonic impedance.

In what follows, we consider maximal efficiency of PA with current waveform (48) (i.e., (50)) and voltage waveform (52) provided that $\left|\underline{z}_{2}\right| \leq 1 /\left|a_{2 i}^{(2 \mathrm{a})}\right|$ and $\operatorname{Re}\left\{\underline{z}_{2}\right\} \geq 0$ in subcase (2a) (i.e., $\left|\underline{z}_{2}\right| \leq 1 /\left|a_{2 i}^{(2 \mathrm{~b})}\right|$ and $\operatorname{Re}\left\{\underline{z}_{2}\right\} \geq 0$ in subcase (2b)). The problem of finding maximal efficiency of PA with waveform pair (52) and (48) (i.e., (50)) for prescribed second-harmonic impedance can be reduced to the problem of finding voltage waveform of type (52) with maximal absolute value of $a_{1 v}$ for prescribed coefficients of the second harmonic [14]. Fourier series expansion of (48) (i.e., (50)) contains only cosine terms and therefore the procedure for the calculation of maximal efficiency of PA described by Algorithm 32 in [14] can be used in both subcases. For the sake of completeness, here we provide Algorithm 3, which is analogous to Algorithm 32 in [14]. We change the notation: $\theta_{0 v}-\xi_{v} / 2$ is replaced with $\varphi_{1 v}$.

In subcase (2a), input of Algorithm 3 (coefficients $a_{1 i}$ and $a_{2 i}$ ) is given by (49). Maximal efficiency of PA with waveform pair (48) and (52), as a function of normalized second-harmonic impedance $\underline{z}_{2 n}=\underline{z}_{2} / \operatorname{Re}\left\{\underline{z}_{1}\right\}$, is presented in Figure 11(a). Efficiency of 0.84 is attained in the vicinity of $\underline{z}_{2 n}=4.89$ (corresponding to $\underline{z}_{2}=4.12$ ).

In subcase (2b), input of Algorithm 3 (coefficients $a_{1 i}$ and $a_{2 i}$ ) is given by (51). Maximal efficiency of PA with waveform pair (50) and (52), as a function of normalized second-harmonic impedance, is presented in Figure 11(b).
Efficiency of 0.87 is achieved in the vicinity of $\underline{z}_{2 n}=37.99$ (corresponding to $\underline{z}_{2}=33.18$ ).

As we mentioned before, the optimal value of parameter $A_{2}$ is $A_{2\left(I_{2}=0\right) \text { cont }}=0.3305$. Corresponding maximal efficiency of inverse class-F PA (see Box 2) is $\eta_{\operatorname{IF}(k=2)}=$ 0.8777 . Current waveform $i^{(2 \mathrm{~b})}(\tau)$ in subcase $(2 \mathrm{~b})$ is close to the optimal for inverse class-F PA and corresponding peak efficiency of 0.87 is close to $\eta_{\mathrm{IF}(k=2)}=0.8777$.

\section{Simulation}

In this section, we provide results of nonlinear simulation of inverse class-F PA based on high performance CGH40010F GaN HEMT, manufactured by Cree Inc. (Section 6.1). Comparison of mathematical models of nonnegative waveforms and intrinsic waveforms of HEMT obtained in simulation is provided in Section 6.2. The example of simulated PA demonstrates that the theoretical results presented in this paper can serve as a useful tool during the design of highefficiency PA.

6.1. Simulation Setup and Results. The circuit diagram of simulated inverse class-F PA is depicted in Figure 12. The proposed design of inverse class-F PA has been implemented in Advanced Design System (ADS). Computations were performed by using a harmonic balance simulator. 


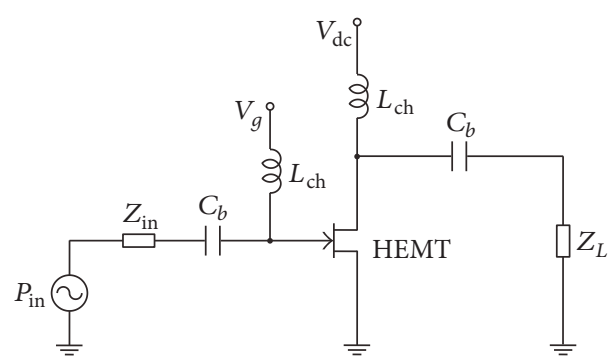

FIGURE 12: Circuit diagram of simulated inverse class-F PA.

The drain and gate biases are set to $V_{\mathrm{dc}}=28 \mathrm{~V}$ and $V_{g}=-1.95 \mathrm{~V}$, respectively. Frequency is set to $f_{0}=$ $900 \mathrm{MHz}\left(\omega_{0}=2 \pi f_{0}\right)$, input power to $P_{\text {in }}=18 \mathrm{dBm}$, capacitance of $\mathrm{dc}$ blocking capacitor to $C_{b}=1 \mu \mathrm{F}$, and inductance of choke to $L_{\mathrm{ch}}=1 \mu \mathrm{H}$.

In simulations of inverse class-F PA, we use large-signal model of CGH40010F provided by the manufacturer Cree Inc. This model allows access to the virtual ports located right at the active device [20]. Thus, it is possible to observe intrinsic waveforms, without the effect of package parasitics [20]. Furthermore, as a part of waveform engineering, to verify the class of operation, it is essential to analyze intrinsic waveforms at current generator plane of the device $[16,20]$. Moreover, such sophisticated model allows designers to seek practical waveforms that approximate theoretically derived waveforms $[16,20]$.

Due to impedance transformation through the output parasitic network of the device, the impedance presented at the current generator plane (intrinsic plane) and load impedance often have considerably different values. To choose the initial values for load impedance at harmonic frequencies up to the third harmonic, we use the approximate equivalent scheme of CGH40010F output parasitic network proposed in [16] (Figure 13). The values of elements of parasitic network are [16] $C_{\mathrm{ds}}=1.22 \mathrm{pF}, L=0.55 \mathrm{nH}$, $C_{p}=0.25 \mathrm{pF}, L_{p}=0.1 \mathrm{nH}$, and $R_{p}=0.1 \Omega$.

Initial values for load impedance are determined from the conditions that values of impedance presented at current generator plane (see Figure 13) at harmonic frequencies are $\underline{Z}\left(\omega_{0}\right)=(70+\mathrm{j} 0) \Omega, \underline{Z}\left(2 \omega_{0}\right)=(700+\mathrm{j} 0) \Omega$, and $\underline{Z}\left(3 \omega_{0}\right)=$ $(0+j 0) \Omega$. By solving these equations numerically, we obtain initial values for load impedance at fundamental and second harmonic: $\underline{Z}_{L}\left(\omega_{0}\right)=\left(48.5+\right.$ j29) $\Omega$ and $\underline{Z}_{L}\left(2 \omega_{0}\right)=(3.8+$ j45.9) $\Omega$. Solving the equation at the third harmonic yields $\underline{Z}_{L}\left(3 \omega_{0}\right)=(-0.2-\mathrm{j} 13.7) \Omega$. Since this value cannot be realized with passive load (real part is negative), for the initial value, we choose $\underline{Z}_{L}\left(3 \omega_{0}\right)=(0-\mathrm{j} 13.7) \Omega$, which transforms to $\underline{Z}\left(3 \omega_{0}\right)=(0.176-\mathrm{j} 0.001) \Omega$ at current generator plane.

The initial value of $Z_{\text {in }}$ (see Figure 12) at fundamental frequency is estimated by using small-signal $S$-parameters provided in the data sheet of CGH40010 [21]. For CGH40010 terminated with $\underline{Z}_{L}\left(\omega_{0}\right)=(48.5+\mathrm{j} 29) \Omega$, by using smallsignal $S$-parameters $\left(900 \mathrm{MHz}, I_{\mathrm{DQ}}=200 \mathrm{~mA}\right)$, we calculate that input impedance of CGH40010 is equal to $(1.1-\mathrm{j} 8.7) \Omega$. Initial value of $\underline{Z}_{\text {in }}\left(\omega_{0}\right)$ is set to complex conjugate value; that is, $\underline{Z}_{\text {in }}\left(\omega_{0}\right)=(1.1+\mathrm{j} 8.7) \Omega$. The initial value of $\underline{Z}_{\text {in }}\left(2 \omega_{0}\right)$ is set to low value, for which we choose $\underline{Z}_{\text {in }}\left(2 \omega_{0}\right)=(1+j 0) \Omega$. For the initial value at the third harmonic, we choose $\underline{Z}_{\text {in }}\left(3 \omega_{0}\right)=$ $(50+j 0) \Omega$.

According to [16], theoretically predicted waveforms provide guidance to the shapes of practical waveforms of PA. During the simulation, we perform waveform engineering approach; that is, we search for target waveforms: the intrinsic current waveform as close as possible to truncated biharmonic waveform and intrinsic voltage waveform as close as possible to optimal voltage waveform with fundamental and second harmonic.

The final values of $Z_{L}$ at fundamental, second, and third harmonic are $\underline{Z}_{L}\left(\omega_{0}\right)=(51.4+\mathrm{j} 31.8) \Omega, \underline{Z}_{L}\left(2 \omega_{0}\right)=(0+$ j65) $\Omega$, and $\underline{Z}_{L}\left(3 \omega_{0}\right)=(0-\mathrm{j} 15) \Omega$. The final values of $Z_{\text {in }}$ at fundamental, second, and third harmonic are $\underline{Z}_{\text {in }}\left(\omega_{0}\right)=$ $(1.9+\mathrm{j} 9.2) \Omega, \underline{Z}_{\text {in }}\left(2 \omega_{0}\right)=(0.5-\mathrm{j} 0.5) \Omega$, and $\underline{Z}_{\text {in }}\left(3 \omega_{0}\right)=(50+$ j0) $\Omega$. The results of simulation are $\eta_{\text {intrinsic }}^{\text {sim }}=0.8746$ intrinsic drain efficiency, $\eta^{\text {sim }}=0.8568$ drain efficiency, and $P_{\text {out }}=$ $39.80 \mathrm{dBm}$ output power at $900 \mathrm{MHz}$.

6.2. Comparison of Waveforms. In this subsection, we provide the comparison of the theoretically predicted waveforms and intrinsic waveforms obtained in simulation. We also compare intrinsic current waveform obtained in simulation with three commonly used models of nonnegative current waveforms.

Simulated intrinsic waveforms $v_{\mathrm{DiSi}}$ and $i_{\mathrm{Di}}$ (see Figure 13 ) can be expressed as follows:

$$
\begin{gathered}
v_{\mathrm{DiSi}}\left(\tau_{s}\right)=V_{0, \mathrm{DiSi}}+\sum_{m=1}^{M} V_{m, \mathrm{DiSi}} \cos \left(m \tau_{s}+\theta_{m, s}\right), \\
i_{\mathrm{Di}}\left(\tau_{s}\right)=I_{0, \mathrm{Di}}+\sum_{m=1}^{M} I_{m, \mathrm{Di}} \cos \left(m \tau_{s}+\psi_{m, s}\right),
\end{gathered}
$$

where $\tau_{s}$ denotes $\omega_{0} t$ and $M$ denotes the order of harmonic balance simulation. After the change of variable $\tau_{s} \rightarrow \tau-\psi_{1, s}$ these waveforms become

$$
\begin{gathered}
v_{\mathrm{DiSi}}(\tau)=V_{0, \mathrm{DiSi}}+\sum_{m=1}^{M} V_{m, \mathrm{DiSi}} \cos \left(m \tau+\theta_{m}\right), \\
i_{\mathrm{Di}}(\tau)=I_{0, \mathrm{Di}}+\sum_{m=1}^{M} I_{m, \mathrm{Di}} \cos \left(m \tau+\psi_{m}\right),
\end{gathered}
$$

where $\theta_{m}=\theta_{m, s}-m \psi_{1, s}$ and $\psi_{m}=\psi_{m, s}-m \psi_{1, s}$. Simulated intrinsic waveforms expressed in form (54) are plotted in Figure 14. Their amplitudes and phases for $m \leq 5$ are presented in Table 2. From this data, it can be concluded that voltage waveform has a significant second harmonic, whereas higher harmonics $(m=3,4,5)$ are small. Furthermore, an almost ideal difference between fundamental and second harmonic phases $\left(\theta_{2}-\theta_{1} \approx 179^{\circ}\right)$ results in the flattening of the bottom of the voltage waveform. 


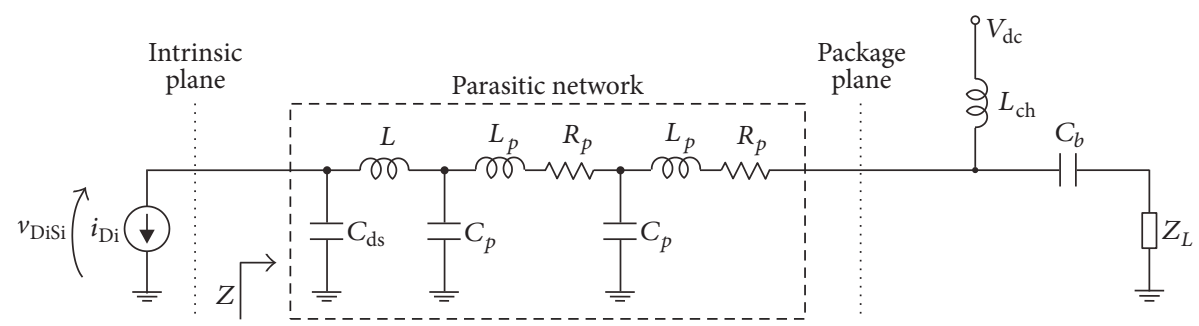

FIGURE 13: Approximate equivalent scheme of CGH40010F output parasitic network [16]. The intrinsic plane (current generator plane) and package plane are marked by a dotted line, and $v_{\mathrm{DiSi}}$ and $i_{\mathrm{Di}}$ denote intrinsic waveforms.

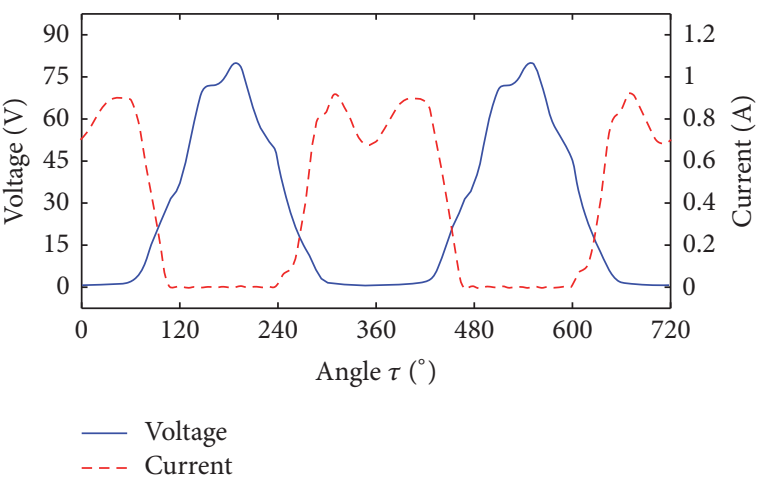

FIGURE 14: Intrinsic waveforms obtained in simulation: $v_{\text {Disi }}$ (solid blue line) and $i_{\mathrm{Di}}$ (dashed red line).

TABLE 2: Frequency-domain data of simulated intrinsic waveforms.

\begin{tabular}{ccccc}
\hline$m$ & $V_{m, \text { DiSi }}[\mathrm{V}]$ & $\theta_{m}\left[^{\circ}\right]$ & $I_{m, \text { Di }}[\mathrm{A}]$ & $\psi_{m}\left[^{\circ}\right]$ \\
\hline 0 & 27.733 & 0.000 & 0.398 & 0.000 \\
1 & 38.369 & -179.883 & 0.503 & 0.000 \\
2 & 11.761 & -0.687 & 0.015 & -94.766 \\
3 & 0.147 & 31.830 & 0.181 & -174.485 \\
4 & 0.349 & 84.385 & 0.059 & -170.941 \\
5 & 0.647 & -121.226 & 0.041 & -17.095 \\
\hline
\end{tabular}

Moreover, the data obtained in simulation are used to calculate impedance presented at the intrinsic plane at each harmonic $(m \geq 1)$ by using the following formula:

$$
\underline{Z}\left(m \omega_{0}\right)=-\frac{V_{m, \mathrm{DiSi}} \mathrm{e}^{\mathrm{j} \theta_{m}}}{I_{m, \mathrm{Di}} \mathrm{e}^{\mathrm{j} \psi_{m}}} .
$$

Values of $\underline{Z}\left(m \omega_{0}\right)$, their magnitude $Z\left(m \omega_{0}\right)$, and arguments $\varphi\left(m \omega_{0}\right)$ for $m \leq 5$ are presented in Table 3. Impedance presented at the intrinsic plane at fundamental frequency has very small imaginary part; it is almost purely real. Furthermore, $Z\left(2 \omega_{0}\right)>10 \cdot Z\left(\omega_{0}\right), Z\left(3 \omega_{0}\right)<Z\left(\omega_{0}\right) / 10$ and
TABLE 3: Values of impedance presented at the intrinsic plane.

\begin{tabular}{lccc}
\hline$m$ & $\underline{Z}\left(m \omega_{0}\right)[\Omega]$ & $Z\left(m \omega_{0}\right)[\Omega]$ & $\varphi\left(m \omega_{0}\right)\left[{ }^{\circ}\right]$ \\
\hline 1 & $76.313+\mathrm{j} 0.156$ & 76.313 & 0.117 \\
2 & $56.926-\mathrm{j} 798.180$ & 800.207 & -85.921 \\
3 & $0.727+\mathrm{j} 0.360$ & 0.811 & 26.315 \\
4 & $1.510+\mathrm{j} 5.765$ & 5.960 & 75.326 \\
5 & $3.892+\mathrm{j} 15.460$ & 15.942 & 75.869 \\
\hline
\end{tabular}

$Z\left(4 \omega_{0}\right)<Z\left(\omega_{0}\right) / 10$. These values show that the magnitude of impedance at the second harmonic is high, while magnitude of impedance at the third and fourth harmonic is low. Magnitude of impedance at the fifth harmonic is $Z\left(5 \omega_{0}\right) \approx$ $Z\left(\omega_{0}\right) / 5$. These values of impedance presented at intrinsic plane confirm inverse class-F mode of operation of simulated PA.

Intrinsic waveforms (54) in normalized form can be written as

$$
\begin{aligned}
v_{\text {DiSinnorm }}(\tau) & =\frac{v_{\text {DiSi }}(\tau)}{V_{0, \mathrm{DiSi}}} \\
& =1+\sum_{m=1}^{M}\left(a_{m \nu} \cos m \tau+b_{m \nu} \sin m \tau\right), \\
i_{\text {Di_norm }}(\tau) & =\frac{i_{\mathrm{Di}}(\tau)}{I_{0, \mathrm{Di}}} \\
& =1+\sum_{m=1}^{M}\left(a_{m i} \cos m \tau+b_{m i} \sin m \tau\right) .
\end{aligned}
$$

Fourier coefficients of normalized waveforms $v_{\text {DiSinnorm }}(\tau)$ and $i_{\text {Di_norm }}(\tau)$ for $m \leq 5$ are presented in Table 4 .

Here, we provide an approximation of simulated intrinsic current waveform with biharmonic truncated waveform of type (6). The normalized intrinsic current waveform is approximated by normalized current waveform of type (6) for $k=2, A_{2}=0.415$, and $\tau_{p}=100^{\circ}$ (conduction angle $\left.2 \tau_{p}=200^{\circ}\right)$, which reads

$$
i^{\bmod }(\tau)= \begin{cases}4.632\left[\cos \tau-\cos 100^{\circ}-0.415\left(\cos 2 \tau-\cos 200^{\circ}\right)\right], & |\tau| \leq 100^{\circ} \\ 0, & 100^{\circ} \leq|\tau| \leq 180^{\circ}\end{cases}
$$




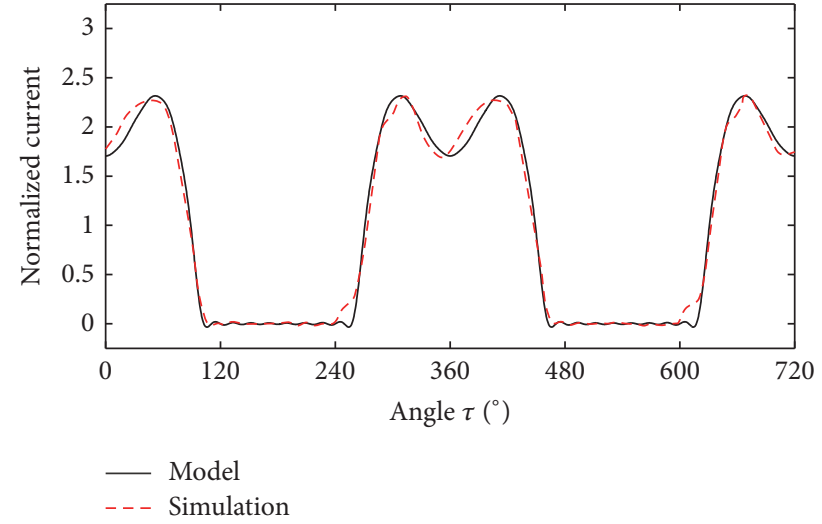

FIGURE 15: Normalized intrinsic current waveform obtained in simulation (dashed red line) and $i^{\bmod }(\tau)$ (solid black line).

TABLE 4: Fourier coefficients of normalized simulated intrinsic waveforms.

\begin{tabular}{ccccc}
\hline$m$ & $a_{m v}$ & $b_{m v}$ & $a_{m i}$ & $b_{m i}$ \\
\hline 0 & 1.0000 & 0.0000 & 1.0000 & 0.0000 \\
1 & -1.3835 & 0.0028 & 1.2643 & 0.0000 \\
2 & 0.4241 & 0.0051 & -0.0031 & 0.0368 \\
3 & 0.0045 & -0.0028 & -0.4542 & 0.0438 \\
4 & 0.0012 & -0.0125 & -0.1456 & 0.0232 \\
5 & -0.0121 & 0.0199 & 0.0975 & 0.0230 \\
\hline
\end{tabular}

Normalized intrinsic current waveforms obtained in simulation (red dashed line) and $i^{\bmod }(\tau)$ (solid black line) are presented in Figure 15. Good agreement between the shapes of the waveforms can be observed. Moreover, basic waveform parameter of simulated waveform (see Table $4 ; \gamma_{I}^{\text {sim }}=a_{1 i}$ ) and basic waveform parameter of $i^{\bmod }(\tau)$ (calculated from (30)) are

$$
\begin{gathered}
\gamma_{I}^{\text {sim }}=1.264, \\
\gamma_{I}^{\text {mod }}=1.267,
\end{gathered}
$$

respectively. Maximal values of current waveforms are also in good agreement.

Next, we compare the efficiency predicted by mathematical model and intrinsic efficiency obtained in simulation. Basic waveform parameter of optimal truncated current waveform for $2 \tau_{p}=200^{\circ}$ (calculated from (31)) is equal to $\gamma_{I, \max }=1.280$ (corresponding to $A_{2}=0.4018$ ). Basic waveform parameter of optimal voltage waveform with $n=2$ (see (34)) is $\gamma_{V, \max }=\sqrt{2}$. Thus, maximal attainable efficiency of inverse class-F for $2 \tau_{p}=200^{\circ}$ is equal to

$$
\eta_{\max }=\frac{1}{2} \gamma_{I, \max } \gamma_{V, \max }=\frac{1}{2} \cdot 1.280 \cdot \sqrt{2}=0.905
$$

See also Box 2. By taking into account knee voltage of HEMT (from the results of simulations, we estimate that $V_{\text {knee }} \approx$ $0.5 \mathrm{~V}), \gamma_{V, \text { max }}$ decreases to

$$
\begin{aligned}
\gamma_{V}^{\text {knee }} & =\gamma_{V, \text { max }} \frac{V_{0, \text { DiSi }}-V_{\text {knee }}}{V_{0, \text { DiSi }}}=\gamma_{V, \text { max }} \cdot 0.98 \\
& =\sqrt{2} \cdot 0.98=1.386,
\end{aligned}
$$

and the corresponding maximal efficiency becomes

$$
\eta_{\max }^{\mathrm{knee}}=\frac{1}{2} \gamma_{I, \max } \gamma_{V, \text { max }} \cdot 0.98=\eta_{\max } \cdot 0.98=0.887 .
$$

In simulation, we obtain that intrinsic drain efficiency is equal to

$$
\eta_{\text {intrinsic }}^{\text {sim }}=\frac{1}{2} \gamma_{I}^{\text {sim }} \gamma_{V}^{\text {sim }}=\frac{1}{2} \cdot 1.264 \cdot 1.384=0.875
$$

(see Table 4; $\gamma_{I}^{\text {sim }}=a_{1 i}$ and $\gamma_{V}^{\text {sim }}=-a_{1 v}$ ). The difference between $\eta_{\max }^{\text {knee }}$ and $\eta_{\text {intrinsic }}^{\text {sim }}$ appears because $\gamma_{I}^{\text {sim }} \approx$ $0.9875 \gamma_{I, \max }$. On the other hand, the efficiency predicted for inverse class-F PA with current waveform (57) and optimal voltage waveform, by taking into account knee voltage, is equal to

$$
\begin{aligned}
\eta_{\text {mod }}^{\text {knee }} & =\frac{1}{2} \gamma_{I}^{\bmod } \gamma_{V, \max } \cdot 0.98=\frac{1}{2} \cdot 1.267 \cdot \sqrt{2} \cdot 0.98 \\
& =0.878 \approx \eta_{\text {intrinsic }} .
\end{aligned}
$$

In what follows, we compare intrinsic current waveform obtained in simulation with the following three commonly used types of nonnegative waveforms:

(i) Rectangular waveform

(ii) Maximally flat odd harmonic waveform

(iii) Odd harmonic waveform with maximal amplitude of fundamental harmonic

All these waveforms are normalized in the sense that $\mathrm{dc}$ component is equal to 1 .

(i) The ideal shape of current waveform for inverse class-F PA is square waveform (rectangular waveform with conduction angle $2 \tau_{p}=180^{\circ}$ ). It is easy to show that the basic waveform parameter of rectangular waveform with conduction angle $2 \tau_{p}$ is equal to

$$
\gamma_{I}^{\text {rect }}\left(\tau_{p}\right)=2 \operatorname{sinc} \tau_{p} .
$$

For $\tau_{p}=100^{\circ}$ and $\tau_{p}=90^{\circ}$, corresponding values are $\gamma_{I}^{\text {rect }}\left(100^{\circ}\right)=1.1285$ and $\gamma_{I}^{\text {rect }}\left(90^{\circ}\right)=\gamma_{I}^{\text {sq }}=4 / \pi \approx 1.273$. Although $\gamma_{I}^{\text {sq }}$ is close to $\gamma_{I}^{\text {sim }}=1.264$, the shape of square waveform (dotted green line in Figure 16) and its maximal value are not in good agreement with the simulated intrinsic current waveform (dashed red line in the same figure).

(ii) Maximally flat odd harmonic waveform [6] denotes nonnegative waveform, which contains dc component, fundamental harmonic, and $q$ consecutive odd harmonics $(q=0$ 


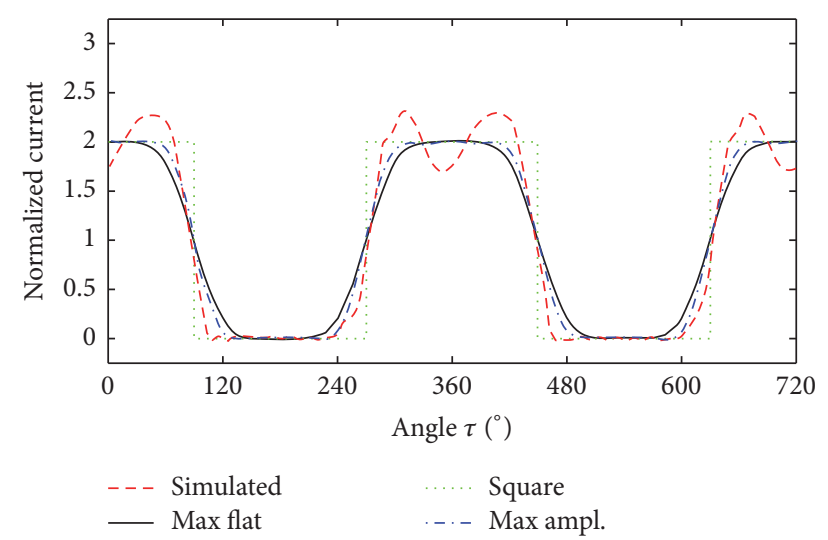

FIGURE 16: Current waveforms: simulated (dashed red line), square (dotted green line), maximally flat odd harmonic for $q=2$ (solid black line) and odd harmonic waveform with maximal fundamental harmonic amplitude for $q=2$ (dash-dotted blue line).

means only fundamental, $q=1$ means fundamental and third harmonic, etc.). Since it is an even function, it contains only cosine terms at fundamental and higher odd harmonics. Maximal value of maximally flat odd harmonic waveform is equal to 2 [6]. The basic waveform parameter of this waveform is equal to [6]

$$
\gamma_{I}^{\max \text { flat }}(q)=2 \frac{((2 q+1) ! !)^{2}}{(2 q) ! !(2 q+2) ! !} .
$$

Corresponding values are $\gamma_{I}^{\max f l a t}(0)=1, \gamma_{I}^{\max f l a t}(1)=$ $9 / 8=1.125, \gamma_{I}^{\max f l a t}(2) \approx 1.172$, and so forth. Parameter $\gamma_{I}^{\max }$ flat $(q)$ slowly increases when $q$ increases and $\gamma_{I}^{\max \text { flat }}(q)<$ $\lim _{q \rightarrow \infty} \gamma_{I}^{\max f l a t}(q)=4 / \pi \approx 1.273$, [6]. Maximally flat odd harmonic waveform tends to square waveform when $q$ tends to infinity [6]. Maximally flat odd harmonic waveform for $q=2$ is plotted in Figure 16 (solid black line).

(iii) Odd harmonic waveform with maximal amplitude of fundamental harmonic contains dc component, fundamental harmonic, and $q$ consecutive odd harmonics. It is an even function and contains only cosine terms. Maximal value of this waveform is also equal to 2. Basic waveform parameter of odd harmonic waveform with maximal amplitude of fundamental harmonic is equal to [10]

$$
\gamma_{I}^{\max \mathrm{ampl}}(q)=\frac{2}{q+2} \cot \left(\frac{\pi}{2(q+2)}\right) .
$$

Corresponding values are $\gamma_{I}^{\operatorname{max~ampl}}(0)=1, \gamma_{I}^{\max \mathrm{ampl}}(1)=$ $2 / \sqrt{3} \approx 1.155, \gamma_{I}^{\max a m p l}(2)=(1+\sqrt{2}) / 2 \approx 1.207$, and so forth. This waveform also tends to square waveform when $q$ tends to infinity. Parameter $\gamma_{I}^{\text {max ampl }}(q)$ increases when $q$ increases and $\gamma_{I}^{\max \text { ampl }}(q)<\lim _{q \rightarrow \infty} \gamma_{I}^{\max \text { ampl }}(q)=4 / \pi \approx$ 1.273. Odd harmonic waveform with maximal amplitude of fundamental harmonic for $q=2$ is plotted in Figure 16 (dashdotted blue line).
TABLE 5: Quantitative indicators of the four considered approximations for intrinsic current waveform. Abbreviations are described in the text.

\begin{tabular}{lcccc}
\hline & Truncated & Square & Max flat & Max ampl. \\
\hline XCORR & $\mathbf{0 . 9 9 5 5}$ & 0.9561 & 0.9737 & 0.9842 \\
R2 & $\mathbf{0 . 9 9 0 3}$ & 0.9071 & 0.9383 & 0.9637 \\
MSE & $\mathbf{0 . 0 0 8 9}$ & 0.0858 & 0.0570 & 0.0336 \\
\hline
\end{tabular}

The above consideration (illustrated in Figures 15 and 16) shows that truncated biharmonic current waveform can provide better approximation for the simulated intrinsic current waveform than other considered types. Additionally, in order to quantify similarities of normalized intrinsic current waveform obtained in simulation with four considered models of nonnegative waveforms, we calculate the following three commonly used quantitative indicators:

(a) Cross-correlation coefficient (denoted as XCORR in Table 5)

(b) R2 statistics or so-called coefficient of determination (denoted as R2 in Table 5)

(c) Mean squared error (denoted as MSE in Table 5)

In Table 5, abbreviation "truncated" denotes waveform (57), "square" denotes normalized square waveform, "max flat" denotes maximally flat odd harmonic waveform for $q=2$, and "max ampl." denotes odd harmonic waveform with maximal fundamental harmonic amplitude for $q=2$. The range of all three indicators is $[0,1]$. Better fit is indicated with XCORR closer to $1, \mathrm{R} 2$ closer to 1 , and MSE closer to 0 . The values of all three indicators confirm that truncated biharmonic current waveform (57) provides better approximation of the simulated intrinsic current waveform in comparison to the other three commonly used types.

\section{Conclusion}

In this paper, we consider two-parameter families of current waveforms of type (1) with $k=2$ or $k=3$. These families include both discontinuous and continuous waveforms. Under common assumptions that the third $(n=3)$ harmonic in current waveform for class-F and the second $(n=2)$ harmonic for inverse class-F are missing, the following four cases are analyzed: (i) $n=k=3$, (ii) $n=3, k=2$, (iii) $n=k=$ 2 , and (iv) $n=2, k=3$. It is shown that in each of these four cases current waveform enabling maximal efficiency (optimal waveform) is continuous for conduction angles listed in Table 1. Furthermore, closed-form expressions for parameters of optimal current waveforms and maximal efficiency of class-F (inverse class-F PA) in terms of conduction angle are provided in Box 1 (Box 2). Two case studies involving suboptimal continuous current waveforms in PA efficiency analysis are also presented. Finally, we provide comparison of the theoretical results derived in this paper with the results obtained in nonlinear simulation of inverse class-F PA based on CGH40010F HEMT. 


\section{Conflicts of Interest}

The authors declare that there are no competing interests regarding the publication of this paper.

\section{Acknowledgments}

The authors wish to thank Cree Inc. for providing large-signal models of GaN HEMTs. They also express their gratitude to Keysight Technologies Inc. for providing them with license for Advanced Design System (ADS) software. This work is supported by the Serbian Ministry of Education, Science and Technology Development as a part of Project TP32016.

\section{References}

[1] F. H. Raab, "Maximum efficiency and output of class-F power amplifiers," IEEE Transactions on Microwave Theory and Techniques, vol. 49, no. 6, pp. 1162-1166, 2001.

[2] Y. Y. Woo, Y. Yang, and B. Kim, "Analysis and experiments for high-efficiency class-F and inverse class-F power amplifiers," IEEE Transactions on Microwave Theory and Techniques, vol. 54, no. 5, pp. 1969-1973, 2006.

[3] J. H. Kim, G. D. Jo, J. H. Oh, Y. H. Kim, K. C. Lee, and J. H. Jung, "Modeling and design methodology of high-efficiency class- $\mathrm{F}$ and class- $\mathrm{F}^{-1}$ power amplifiers," IEEE Transactions on Microwave Theory and Techniques, vol. 59, no. 1, pp. 153-165, 2011.

[4] J. Moon, S. Jee, J. Kim, J. Kim, and B. Kim, "Behaviors of class$\mathrm{F}$ and class- $\mathrm{F}^{-1}$ amplifiers," IEEE Transactions on Microwave Theory and Techniques, vol. 60, no. 6, pp. 1937-1951, 2012.

[5] A. Grebennikov, N. O. Sokal, and M. J. Franco, Switchmode RF Power Amplifiers, Elsevier/Academic Press, San Diego, Calif, USA, 2nd edition, 2012.

[6] A. Juhas and L. A. Novak, "Maximally flat waveforms with finite number of harmonics in class-F power amplifiers," Mathematical Problems in Engineering, vol. 2013, Article ID 169590, 9 pages, 2013.

[7] S. Iguchi, A. Saito, K. Watanabe, T. Sakurai, and M. Takamiya, "Design method of class-F power amplifier with output power of-20 dBm and efficient dual supply voltage transmitter," IEEE Transactions on Circuits and Systems I: Regular Papers, vol. 61, no. 10, pp. 2978-2986, 2014.

[8] A. Juhas and L. A. Novak, "Closed form of optimal current waveform for class-F PA up to fourth harmonic," SadhanaAcademy Proceedings in Engineering Sciences, vol. 40, no. 2, pp. 425-436, 2015.

[9] F. H. Raab, "Class-E, class-C, and class-F power amplifiers based upon a finite number of harmonics," IEEE Transactions on Microwave Theory and Techniques, vol. 49, no. 8, pp. 1462-1468, 2001.

[10] J. D. Rhodes, "Output universality in maximum efficiency linear power amplifiers," International Journal of Circuit Theory and Applications, vol. 31, no. 4, pp. 385-405, 2003.

[11] M. Roberg and Z. Popović, "Analysis of high-efficiency power amplifiers with arbitrary output harmonic terminations," IEEE Transactions on Microwave Theory and Techniques, vol. 59, no. 8, pp. 2037-2048, 2011.

[12] T. Canning, P. J. Tasker, and S. C. Cripps, "Continuous mode power amplifier design using harmonic clipping contours: theory and practice," IEEE Transactions on Microwave Theory and Techniques, vol. 62, no. 1, pp. 100-110, 2014.

[13] A. Juhas and L. A. Novak, "General description of nonnegative waveforms up to second harmonic for power amplifier modelling," Mathematical Problems in Engineering, vol. 2014, Article ID 709762, 18 pages, 2014.

[14] A. Juhas and L. A. Novak, "Conflict set and waveform modelling for power amplifier design," Mathematical Problems in Engineering, vol. 2015, Article ID 585962, 29 pages, 2015.

[15] P. J. Tasker, “Practical waveform engineering," IEEE Microwave Magazine, vol. 10, no. 7, pp. 65-76, 2009.

[16] P. J. Tasker and J. Benedikt, "Waveform inspired models and the harmonic balance emulator," IEEE Microwave Magazine, vol. 12, no. 2, pp. 38-54, 2011.

[17] N. S. Fuzik, "Biharmonic modes of a tuned RF power amplifier," Radiotehnika, vol. 25, no. 7, pp. 62-71, 1970 (Russian).

[18] P. Colantonio, F. Giannini, G. Leuzzi, and E. Limiti, "Theoretical facet and experimental results of harmonic tuned PAs," International Journal of $R F$ and Microwave Computer-Aided Engineering, vol. 13, no. 6, pp. 459-472, 2003.

[19] T. Sharma, R. Darraji, F. Ghannouchi, and N. Dawar, "Generalized continuous class-F harmonic tuned power amplifiers," IEEE Microwave and Wireless Components Letters, vol. 26, no. 3, pp. 213-215, 2016.

[20] R. S. Pengelly, W. Pribble, and T. Smith, "Inverse class-F design using dynamic loadline GaN HEMT models to help designers optimize PA efficiency (Application Note)," IEEE Microwave Magazine, vol. 15, no. 6, pp. 134-147, 2014.

[21] CGH40010 Rev 4.0, Data Sheet, http://www.wolfspeed.com/rf. 


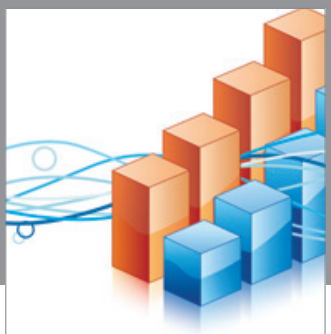

Advances in

Operations Research

vatem alat4

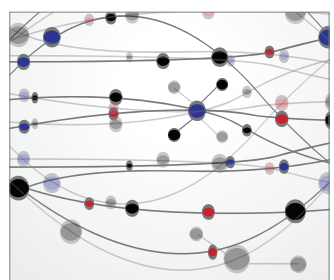

\section{The Scientific} World Journal
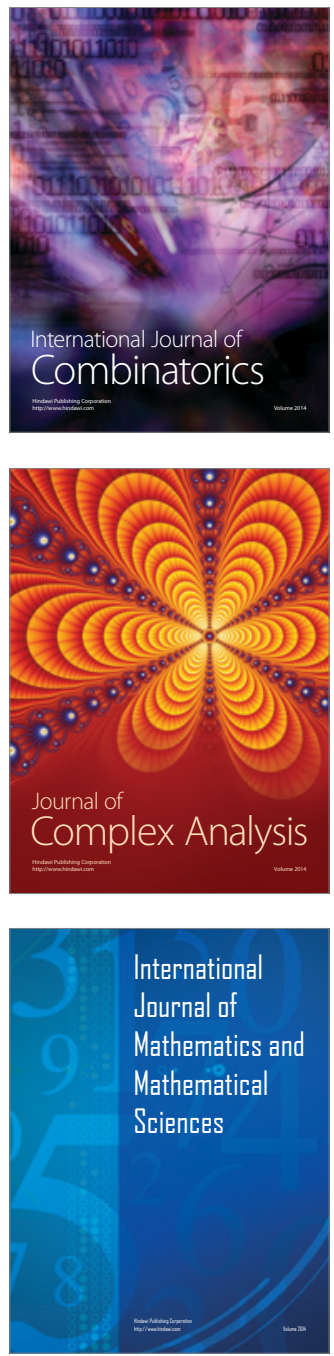
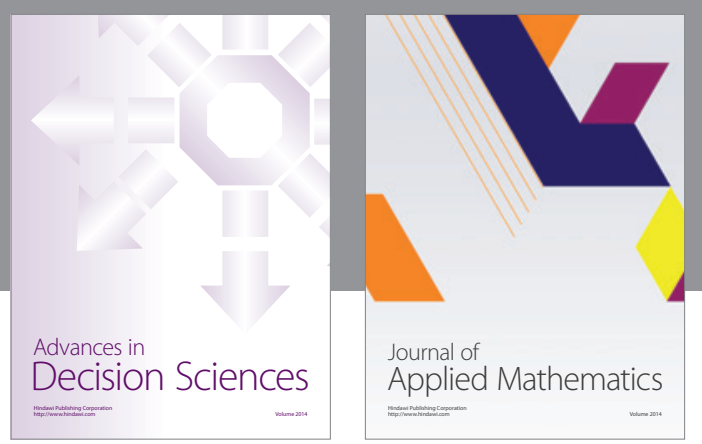

Algebra

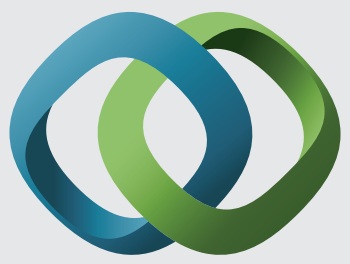

\section{Hindawi}

Submit your manuscripts at

https://www.hindawi.com
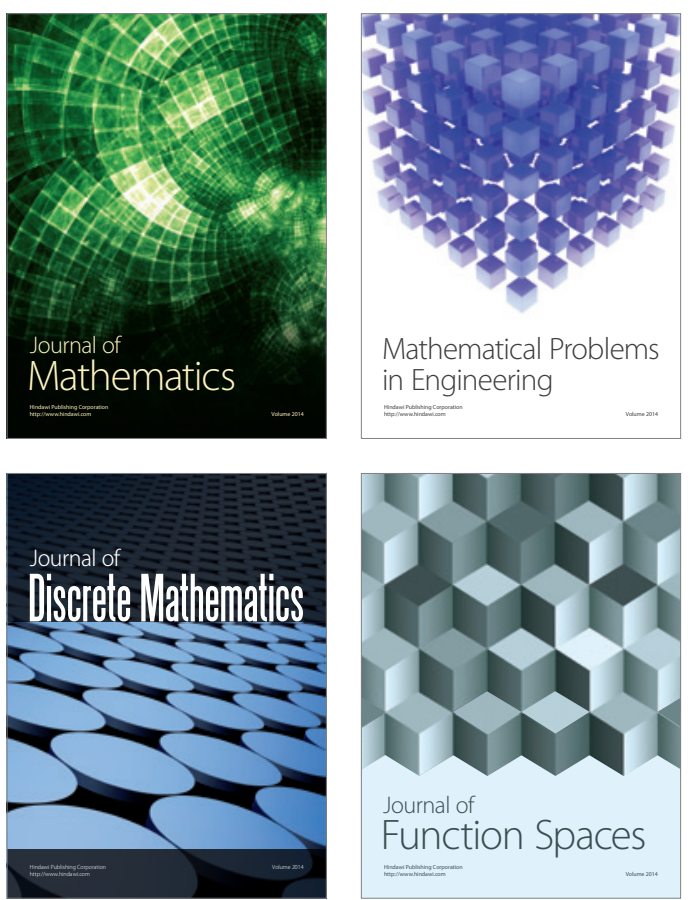

Mathematical Problems in Engineering
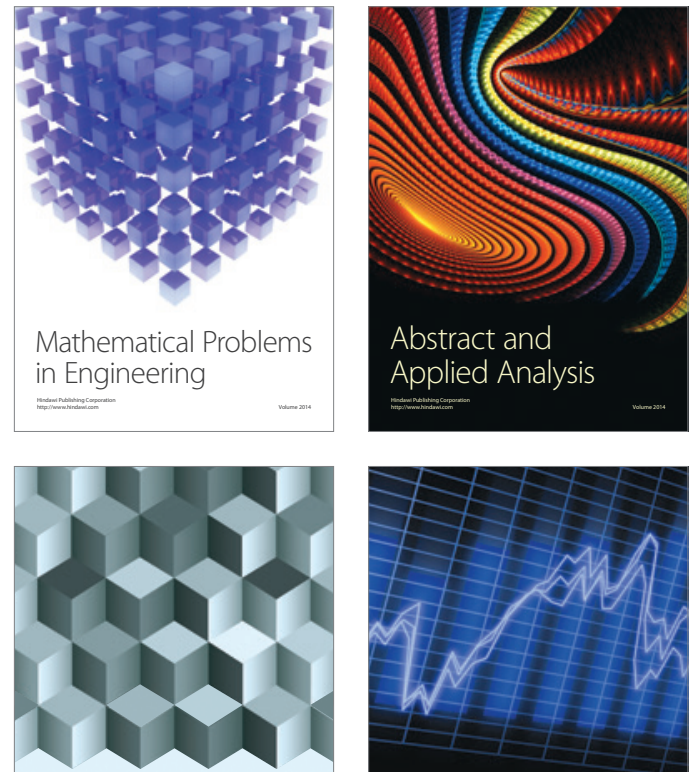

Journal of

Function Spaces

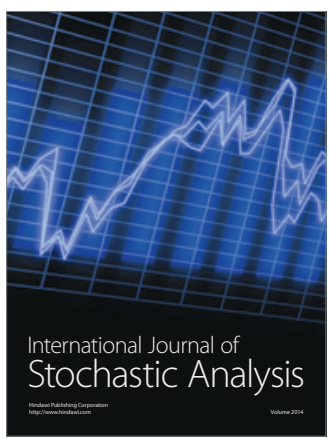

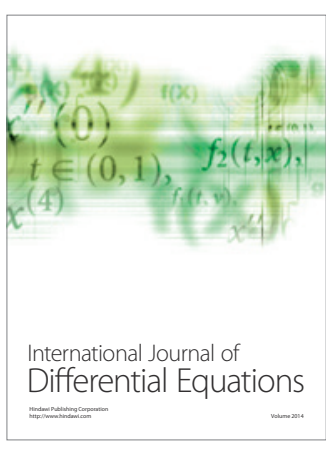
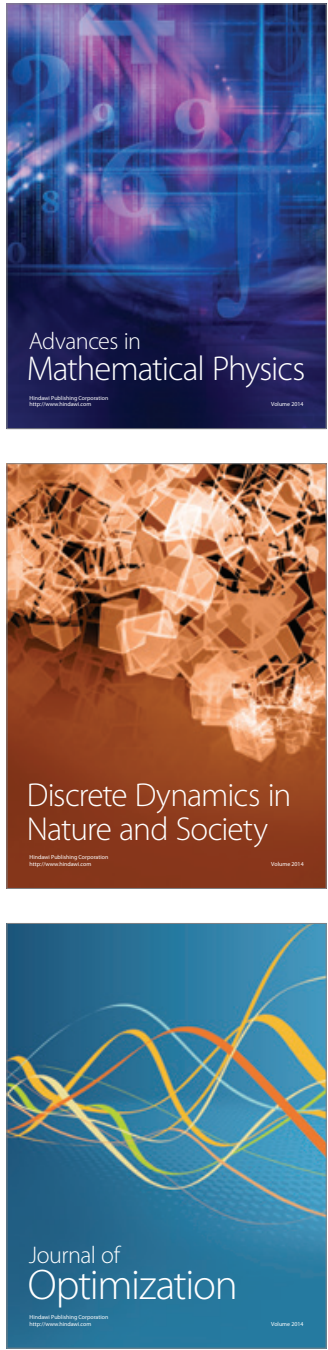